\title{
Oak Ridge National Laboratory Summary of CTF Modeling and Numerical Improvements for Boiling Water Reactor Simulation
}

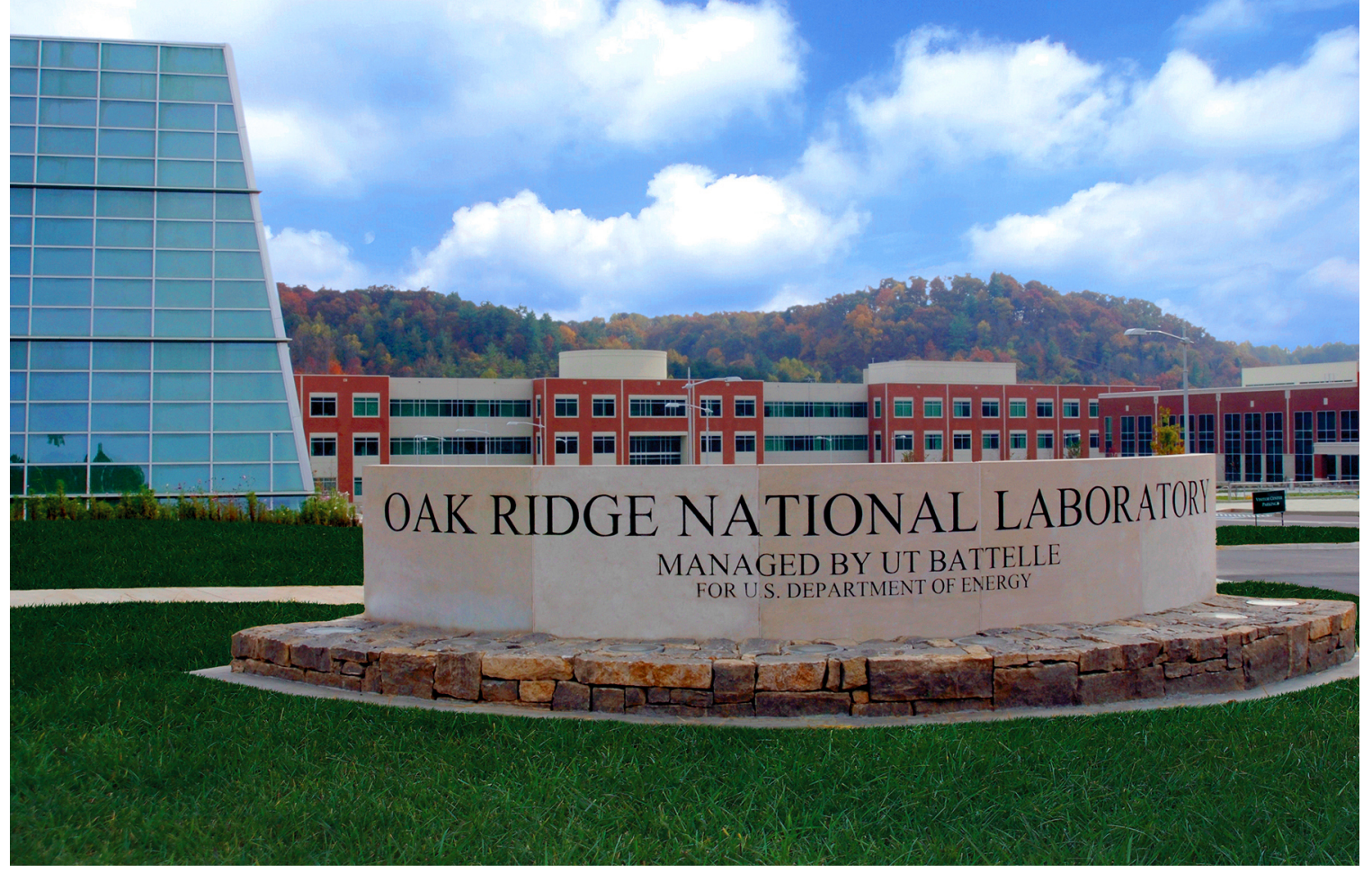

\section{R. Salko \\ B. Hizoum \\ A. Graham \\ B. Collins \\ M. Asgari}

April 30, 2021
Approved for public release. Distribution is unlimited. 


\section{DOCUMENT AVAILABILITY}

Reports produced after January 1, 1996, are generally available free via US Department of Energy (DOE) SciTech Connect.

Website: www.osti.gov/

Reports produced before January 1, 1996, may be purchased by members of the public from the following source:

National Technical Information Service

5285 Port Royal Road

Springfield, VA 22161

Telephone: 703-605-6000 (1-800-553-6847)

TDD: $703-487-4639$

Fax: 703-605-6900

E-mail: info@ntis.gov

Website: http://classic.ntis.gov/

Reports are available to DOE employees, DOE contractors, Energy Technology Data Exchange representatives, and International Nuclear Information System representatives from the following source:

Office of Scientific and Technical Information

PO Box 62

Oak Ridge, TN 37831

Telephone: 865-576-8401

Fax: 865-576-5728

E-mail: report@osti.gov

Website: http://www.osti.gov/contact.html

This report was prepared as an account of work sponsored by an agency of the United States Government. Neither the United States Government nor any agency thereof, nor any of their employees, makes any warranty, express or implied, or assumes any legal liability or responsibility for the accuracy, completeness, or usefulness of any information, apparatus, product, or process disclosed, or represents that its use would not infringe privately owned rights. Reference herein to any specific commercial product, process, or service by trade name, trademark, manufacturer, or otherwise, does not necessarily constitute or imply its endorsement, recommendation, or favoring by the United States Government or any agency thereof. The views and opinions of authors expressed herein do not necessarily state or reflect those of the United States Government or any agency thereof. 
ORNL/TM-2021/2004

\author{
Nuclear Energy and Fuel Cycle Division
}

\title{
Summary of CTF Modeling and Numerical Improvements for Boiling Water Reactor Simulation
}
Author(s)
R. Salko
B. Hizoum
A. Graham
B. Collins
M. Asgari

Date Published: April 30, 2021

\author{
Prepared by \\ OAK RIDGE NATIONAL LABORATORY \\ Oak Ridge, TN 37831-6283 \\ managed by \\ UT-Battelle LLC \\ for the \\ US DEPARTMENT OF ENERGY \\ under contract DE-AC05-00OR22725
}





\section{CONTENTS}

LIST OF FIGURES $\ldots \ldots \ldots \ldots \ldots \ldots \ldots \ldots \ldots \ldots \ldots$

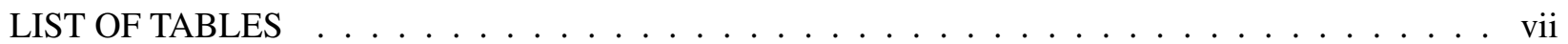

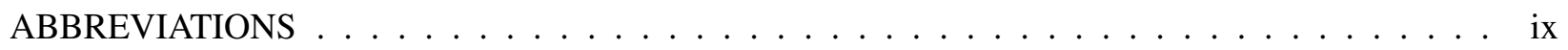

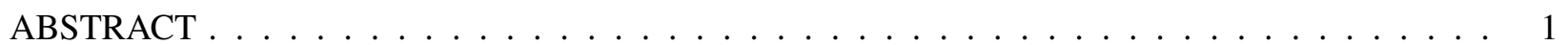

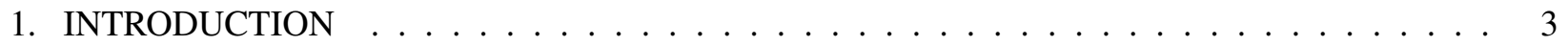

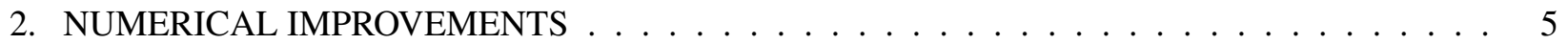

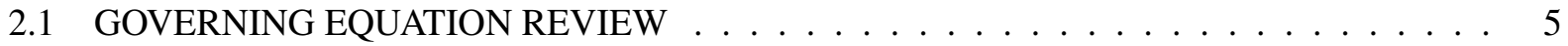

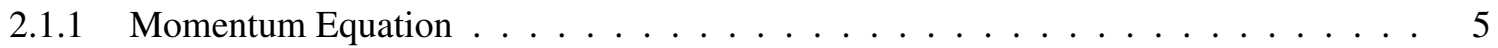

2.1.2 Improved Interfacial Shear Linearization _ . . . . . . . . . . . . . . . 6

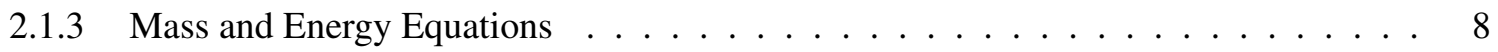

2.1 .4 Inertial Term . . . . . . . . . . . . . . . . . . . . . . . . 9

2.1.5 Linearization Improvements to Advection Terms . . . . . . . . . . . . . . . 11

2.2 REVIEW OF TIME STEPPING LOGIC . . . . . . . . . . . . . . . . . . . . 13

2.3 PARALLELIZATION IMPROVEMENTS . . . . . . . . . . . . . . . . . . 16

3. GEOMETRY MODELING IMPROVEMENTS . . . . . . . . . . . . . . . . . . . . . . . . . 19

3.1 NONUNIFORM CORES . . . . . . . . . . . . . . . . . . . . . . . . . . 19

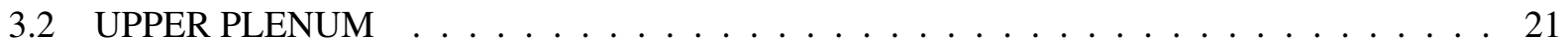

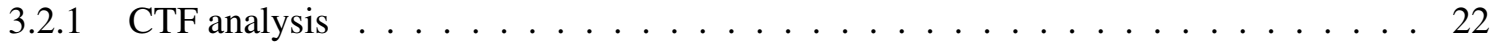

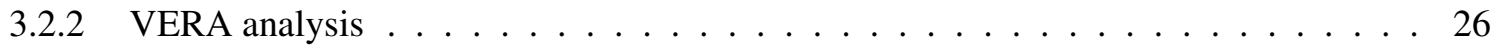

3.3 GRID LOSS TREATMENT REVIEW . . . . . . . . . . . . . . . . . . . 31

3.4 LOWER TIE PLATE FORM LOSS . . . . . . . . . . . . . . . . . . . . 32

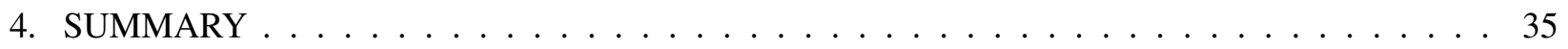

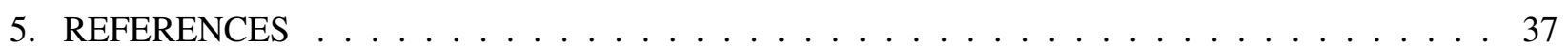





\section{LIST OF FIGURES}

1 Average percentage increase in maximum timestep size in the solution for five selected validation test categories when switching from the original Courant-Friedrichs-Lewy approach to the new one. . . . . . . . . . . . . . . . . . 15

2 Nonuniform region of PSBT axial mesh (not to scale) that causes the original and new Courant-Friedrichs-Lewy implementation to predict different timestep sizes. . . . . . . . . 16

3 Domain decomposition of a GE-14 assembly with different MPI domains denoted by color. . 17

4 Performance improvement of various sections of CTF when increasing the number of processors from 1 to 4 for the GE-14 single-assembly case. Percentage of time spent in each code section (for the 4-processor case) is shown below the section heading. . . . . . . . 18

5 Example of the new model map input format using BWRs in the CTF input deck. . . . . . 20

6 Outlet radial void distribution in a mini-core BWR model which includes mixed

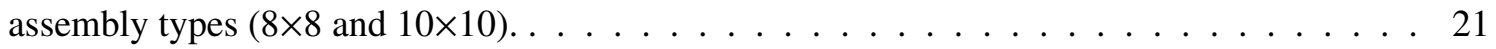

7 Subchannel cross sectional flow areas in the last heated level and upper plenum region of the core for four selected fuel assemblies. . . . . . . . . . . . . . . . . . 23

8 Axial cutaway of the core made through row 5 of subchannels in the assembly lattice. The cutaway is shown for six selected assemblies and denotes how the cross sectional area of the subchannels changes along the axial direction because of part-length rods and axial varying water rods. . . . . . . . . . . . . . . . . . . . 24

9 Quality distribution compared between adjacent levels. . . . . . . . . . . . . . . . 25

10 The difference in power distribution between a model of Peach Bottom with the upper plenum compared to a model without the upper plenum. . . . . . . . . . . . 26

11 The difference in the outlet void distribution between a model of Peach Bottom with the upper plenum and one without the upper plenum. . . . . . . . . . . . . 27

12 The difference in the void distribution between a model of Peach Bottom with the upper plenum and one without the upper plenum. Results are shown for the $309.64 \mathrm{~cm}$ axial location. . . . . . . . . . . . . . . . . . . . 28

13 The difference in the inlet mass flux distribution between a model of Peach Bottom with the upper plenum and one without the upper plenum. . . . . . . . . . . . . . . . 29

14 The difference in the inlet pressure distribution between a model of Peach Bottom with the upper plenum and one without the upper plenum. . . . . . . . . . . . 30

15 Calculated flow distribution in the first level of the tie plate verification test. . . . . . . . 33 



\section{LIST OF TABLES}

1 Summary of activities required for improvement of CTF modeling of BWRs . . . . . . . . 4

2 Summary of fully implicit discretization derivation, implementation, and testing

Momentum Equation . . . . . . . . . . . . . . . . . . . 8

3 Summary of fully implicit discretization derivation, implementation, and testing of mass

and energy Equations. . . . . . . . . . . . . . . . . . . . . 9

4 Summary of performance improvement when solving a single GE-14 assembly using

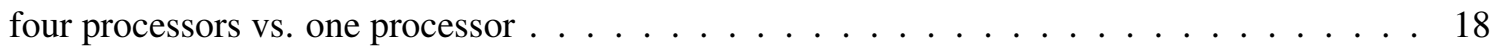

5 Summary of performance improvement when solving a quarter-symmetry Peach Bottom Unit 2 model using 4 processors vs. 1 processor . . . . . . . . . . . . . . . . . . . 19

6 Geometry details for the lower tie plate form loss verification test . . . . . . . . . . 33

7 Analytical calculation of the pressure drop breakdown in the lower tie plate verification test 34 



\section{ABBREVIATIONS}

1D one-dimensional

2D two-dimensional

3D three-dimensional

BWR boiling water reactor

CASL Consortium for Advanced Simulation of Light Water Reactors

CFL Courant-Friedrichs-Lewy

CPR critical power ratio

DOE US Department of Energy

EOHL end of heated length

FOA funding opportunity announcement

INL Idaho National Laboratory

MPI Message Passing Interface

PETSc Portable, Extensible Toolkit for Scientific Computation

PSBT PWR Subchannel and Bundle Tests

PWR pressurized water reactor

SG spacer grid

T/H thermal hydraulics

VERA Virtual Environment for Reactor Applications 



\begin{abstract}
This report documents geometry and numerical improvements made to CTF for the modeling of boiling water reactor (BWR) geometry and operating conditions. These activities are part of a larger program to extend the Virtual Environment for Reactor Applications (VERA) to better support BWR modeling and simulation. The activities documented in this report added features to CTF, including support for mixed-fuel cores, modeling of the upper plenum, and modeling of the lower tie plate form losses. A review of the spacer grid modeling approach was also performed, and a plan was discussed for future improvement. The parallelization of the model was improved, leading to a roughly $2 \times$ improvement in CTF runtime and a 1.6× improvement in total VERA runtime. An in-depth review of the governing equations and their linearization was performed and is documented in this report. Once implemented, this new linearization will allow CTF to take much larger timesteps, leading to more significant reductions in CTF and VERA runtimes.
\end{abstract}





\section{INTRODUCTION}

As part of a two-year US Department of Energy (DOE) funding opportunity announcement (FOA), the Virtual Environment for Reactor Applications (VERA) is being expanded to support modeling and simulation of boiling water reactor (BWR) geometry and operating conditions. CTF is the subchannel capability that is used for thermal hydraulics $(\mathrm{T} / \mathrm{H})$ feedback in VERA. During Consortium for Advanced Simulation of Light Water Reactors (CASL) development work, several activities were identified that were required to improve the capabilities of $\mathrm{CTF}$ for BWR modeling. A preliminary report was released that documented several of the CTF needs for BWR modeling. The requirements have evolved over the course of the project, resulting in the activities list presented in Table 1 . Note that the various activities are supported by multiple projects but are all shown in this table to present a comprehensive picture of the work being done to improve CTF for BWRs. The table is organized into three primary sections, including geometry improvements, improvements to modeling accuracy, and improvements to the numerical stability and performance of the code. Items addressed during this milestone effort are shown in italics; the report section number describing that activity is provided in the "Documentation" column. The "Documentation" column includes references to documents describing past efforts. Ongoing activities to be delivered later in the fiscal year are labeled "Report pending" in the "Documentation" column. The check marks in the "Status" column are color coded as follows: black means the work is finished, green means it was addressed but more work will be required in the future, and blue means it is a pending activity to be completed later in the fiscal year. 
Table 1. Summary of activities required for improvement of CTF modeling of BWRs

\begin{tabular}{|c|c|c|}
\hline Activity & Status & Documentation \\
\hline \multicolumn{3}{|l|}{ Geometry improvements } \\
\hline Addition of inlet orifice map & $\checkmark$ & Salko et al. [2020a] \\
\hline Support for large channel box radii & $\checkmark$ & Salko et al. [2020a] \\
\hline Addition of support for axially varying water rods & $\checkmark$ & Salko et al. [2020a] \\
\hline Support for mixed fuel cores & $\checkmark$ & 3.1 \\
\hline Modeling of upper plenum & $\checkmark$ & 3.2 \\
\hline Two-phase form loss model & $\checkmark$ & 3.3 \\
\hline Lower tie-plate form loss model & $\checkmark$ & 3.4 \\
\hline Bypass modeling & $\checkmark$ & Report pending \\
\hline Part-length rods & $\checkmark$ & Salko and Kumar [2020] \\
\hline Quarter symmetry support & $\checkmark$ & Salko et al. [2020a] \\
\hline \multicolumn{3}{|l|}{ Modeling improvements } \\
\hline Bubbly flow drift flux model & $\checkmark$ & Salko et al. [2020b] \\
\hline Subcooled boiling model & $\checkmark$ & Salko et al. [2020b] \\
\hline Closure model calibration demonstration & $\checkmark$ & Salko et al. [2020b] \\
\hline Drift flux and wall shear calibration & $\checkmark$ & Report pending \\
\hline Inclusion of FRIGG data in validation matrix & $\checkmark$ & Report pending \\
\hline Expansion of Riso validation tests & $\checkmark$ & Report pending \\
\hline Assessment of void drift and turbulent mixing & $\checkmark$ & Report pending \\
\hline Wall shear model & $\checkmark$ & Report pending \\
\hline Flow regime map & $\checkmark$ & Report pending \\
\hline Annular mist interfacial drag assessment & $\checkmark$ & Report pending \\
\hline Wall heat transfer review & $\checkmark$ & Report pending \\
\hline Improvements to two-phase validation matrix infrastructure & $\checkmark$ & Salko and Kumar [2020] \\
\hline Addition of boiling validation tests to validation matrix & $\checkmark$ & Salko and Kumar [2020] \\
\hline \multicolumn{3}{|l|}{ Numerical improvements } \\
\hline Outer iteration loop & $\checkmark$ & Salko et al. [2020a] \\
\hline Governing equation linearization & $\checkmark$ & 2.1 \\
\hline Timestep review and improvement & $\checkmark$ & 2.2 \\
\hline Parallelization improvements & $\checkmark$ & 2.3 \\
\hline Pressure balance loop performance improvements & $\checkmark$ & Salko et al. [2020a] \\
\hline Solver improvements & $\checkmark$ & Salko et al. [2020a] \\
\hline
\end{tabular}




\section{NUMERICAL IMPROVEMENTS}

\subsection{GOVERNING EQUATION REVIEW}

The CTF transient analysis code is based on a two-fluid, three-field (i.e., vapor [v], continuous liquid [ $\ell$ ], and entrained liquid droplets [e]) modeling approach within the subchannels in three-dimensional (3D) Cartesian coordinates. Three sets of one-dimensional (1D) mass equations are solved, along with two sets of energy equations: one for liquid and droplets, and one for vapor. Three sets of momentum equations are solved for both axial and lateral directions. In addition, the code can calculate the mass of the noncondensable gases. Thus, the model features nine balance equations. The corresponding main variables are the three phasic mass flow rates $\left(\dot{m}_{k}\right)$ originating from expressing the momentum equation in conservative form, the two phasic enthalpies, $\alpha_{v} h_{v}$ and $\left(1-\alpha_{\ell}\right) h_{\ell}$; one common pressure $(\mathrm{P})$ with noncondensable partial pressure $\left(P_{a}\right)$; and two volume fractions, $\left(\alpha_{v}\right)$ and $\left(\alpha_{\ell}\right)$. The computational scheme of the code is constructed on the basis of the semi-implicit method: it solves the two-phase, multi-field, time-dependent governing equations in both axial and lateral directions, along with models for turbulent mixing and void drift to calculate the void distribution across the bundle subchannels, droplet deposition, and film entrainment modeling to determine the subchannel local $\mathrm{T} / \mathrm{H}$ conditions. CTF performs a transient solution of the governing equations, and a pseudo transient is solved when performing a steady-state solution.

During a coupled VERA simulation, roughly $50 \%$ of the walltime (real time of the simulation) is spent in CTF for pressurized water reactor (PWR) simulations; however, for BWR simulations, this number is closer to $80 \%$ because of the stricter timestep requirements for two-phase flow, as well as solution of the outer pressure balance loop that is used for determining assembly inlet flow distribution. For certain simulations, the timestep must be maintained below $1 \times 10^{-4} \mathrm{~s}$ to allow for the code to remain numerically stable and to allow it to reach steady state. This behavior is an indication of the use of explicit discretization on the conservation equations. To investigate, an assessment of the full momentum, mass, and energy solution algorithm was performed to determine the necessary steps needed to relax the constraints on timestep size and hence reduce overall simulation runtime. This section presents the results from the review of the discretized momentum and energy equations. A high-level overview of the terms that have been reviewed and their current development status is provided in Table 2.

\subsubsection{Momentum Equation}

Review of the CTF momentum discretization revealed that the equation uses a mixture of explicit and implicit finite difference schemes to linearize the equation. The fully implicit scheme is carried out only for the inertial term and the momentum due to evaporation, which is negligible anyway. A fully explicit discretization is employed for the advection flux and mixing, but partially implicit discretization is employed for terms that involve pressure propagation, such as interfacial/wall shear, as well as grid spacer form loss. This type of discretization limits the timestep and can cause some stability problems. The goal of the CTF numerical improvements is to enhance the semi-implicit method by treating the convection terms, as well as the source terms in the momentum equation, implicitly. This allows the code to violate the Courant-Friedrichs-Lewy (CFL) limit and helps reduce the overall runtime for a core simulation of a slow transient or steady-state conditions. For this phase of work, only the interfacial shear and wall shear are discussed; improvement of the linearization to the advection and mixing will be part of the future work. 


\subsubsection{Improved Interfacial Shear Linearization}

The CTF Theory Manual Salko et al. [2017] describes the interfacial drag model in detail. In this model, the interfacial shear is expressed as a coefficient multiplied by a relative velocity between phases. This differs in form from the drag force equation in that the squared velocity was split into an explicit velocity term multiplied by an implicit velocity term. The implicit term is solved during the momentum equation solution, whereas the explicit velocity is absorbed into the interfacial drag coefficient. The form of the equation is shown in Eq. (1), where the $k_{v \ell, x}$ term is the interfacial shear coefficient that absorbs the explicit relative velocity. The $\bar{V}_{r}^{n+1}$ term is the implicit relative velocity between phases.

$$
\tau_{i_{l v}}=k_{v_{\ell, x}} \bar{V}_{r}^{n+1}
$$

This solution procedure relies on the finite difference motion equations containing no more than the first power of the new-time velocity. This permits a direct solution for $\bar{V}_{r}^{n+1}$ as a linear function of adjacent new-time velocity; however, this can cause stability problems. To improve CTF numerical stability, a fully implicit linearization of the interfacial shear term must be performed as follows,

$$
\left(\tau_{i_{v}}\right)_{i, j+\frac{1}{2}}^{n+1}=\bar{k}_{v \ell, x}\left|\bar{V}_{r}^{n+1}\right| \bar{V}_{r}^{n+1}
$$

where subscript (i) is the subchannel number in the lateral direction, and $\left(j+\frac{1}{2}\right)$ is the interface number between axial cell (j) and $(\mathrm{j}+1) \cdot \bar{V}_{r}^{n+1}$ is defined as the relative phasic velocity, which in bubbly churn flow is defined as $\bar{V}_{r}^{n+1}=\left(v_{v_{i, j+\frac{1}{2}}}^{n+1}-v_{\ell_{i, j+\frac{1}{2}}^{n}}^{n}\right)$ for the CTF original interfacial shear formulation. Note that the drag coefficient, $\bar{k}_{v \ell, x}$, is the same as $k_{v \ell, x}$ but without the explicit part of the relative velocity. Using the Newton linearization, Eq. (2) can be written as follows:

$$
\begin{aligned}
\left(\tau_{i_{\ell v}}\right)_{i, j+\frac{1}{2}}^{n+1}=\left(\tau_{i_{\ell v}}\right)_{i, j+\frac{1}{2}}^{n} & +\left(\frac{\partial \tau_{i_{\ell v}}}{\partial v_{v}}\right)_{i, j+\frac{1}{2}}^{n}\left(v_{v_{i, j+\frac{1}{2}}}^{n+1}-v_{v_{i, j+\frac{1}{2}}}^{n}\right) \\
& +\left(\frac{\partial \tau_{i_{\ell v}}}{\partial v_{\ell}}\right)_{i, j+\frac{1}{2}}^{n}\left(v_{\ell_{i, j+\frac{1}{2}}}^{n+1}-v_{\ell_{i, j+\frac{1}{2}}}^{n}\right) .
\end{aligned}
$$

The derivatives are evaluated as

$$
\begin{aligned}
& \left(\frac{\partial \tau_{i_{\ell v}}}{\partial v_{v}}\right)_{i, j+\frac{1}{2}}^{n}=+2 \bar{k}_{v \ell, x}\left|v_{v_{i, j+\frac{1}{2}}}^{n}-v_{\ell_{i, j+\frac{1}{2}}}^{n}\right| \\
& \left(\frac{\partial \tau_{i_{\ell v}}}{\partial v_{\ell}}\right)_{i+\frac{1}{2}, j}^{n}=-2 \bar{k}_{v \ell, x}\left|v_{\ell_{i, j+\frac{1}{2}}}^{n}-v_{\ell_{i, j+\frac{1}{2}}^{n}}^{n}\right|
\end{aligned}
$$

and the interfacial shear at the old timestep is given by 


$$
\left(\tau_{i, l v}\right)_{i+\frac{1}{2}, j}^{n}=\bar{k}_{v_{\ell, x}}\left|v_{v_{i+\frac{1}{2}, j}}^{n}-v_{\ell_{i+\frac{1}{2}, j}}^{n}\right|\left(v_{v_{i+\frac{1}{2}, j}}^{n}-v_{\ell_{i+\frac{1}{2}, j}}^{n}\right) .
$$

Substituting Eqs. (4) and (5) into Eq. (3) and rearranging this equation with respect to phase velocity at time level $(n)$ and $(n+1)$ leads to the following:

$$
\begin{aligned}
\left(\tau_{i_{\ell v}}\right)_{i, j+\frac{1}{2}}^{n+1} & =-\bar{k}_{v \ell, x}\left|v_{v_{i, j+\frac{1}{2}}}^{n}-v_{\ell_{i+\frac{1}{2}, j}^{n}}\right|\left(v_{v_{i, j+\frac{1}{2}}}^{n}-v_{\ell_{i+\frac{1}{2}, j}}^{n}\right) \\
& +2 \bar{k}_{v \ell, x}\left|v_{v_{i, j+\frac{1}{2}}}^{n}-v_{\ell_{i, j+\frac{1}{2}}}^{n}\right|\left(v_{v_{i, j+\frac{1}{2}}^{n}}^{n+1}-v_{\ell_{i, j+\frac{1}{2}}^{n+1}}\right) .
\end{aligned}
$$

Replacing $k_{v \ell, x}=\bar{k}_{v \ell, x}\left|v_{v_{i, j+\frac{1}{2}}}^{n}-v_{\ell_{i, j+\frac{1}{2}}^{n}}^{n}\right|$ in the equation above and simplifying it gives

$$
\begin{aligned}
\left(\tau_{i_{\ell v}}\right)_{i, j+\frac{1}{2}}^{n+1} & =-k_{v \ell, x}\left(v_{v_{i, j+\frac{1}{2}}^{n}}^{n}-v_{\ell_{i, j+\frac{1}{2}}}^{n}\right) \\
& +2 k_{v \ell, x}\left(v_{v_{i, j+\frac{1}{2}}^{n+1}}^{n+1}-v_{\ell_{i+\frac{1}{2}, j}^{n+1}}^{n+1}\right) .
\end{aligned}
$$

A similar procedure can be followed to derive the fully implicit wall shear, which will lead to

$$
\left(\tau_{w}\right)_{i, j+\frac{1}{2}}^{n+1}=k_{w}\left(2 \dot{m}_{k_{i, j+\frac{1}{2}}^{n+1}}^{n+\dot{m}_{k_{i, j+\frac{1}{2}}}^{n}}\right) \Delta X
$$

For the spacer grid (SG) loss, the linearization is as follows:

$$
\left(\tau_{\text {grid }}\right)_{i, j+\frac{1}{2}}^{n+1}=K_{\text {grid }}\left(2 \dot{m}_{k_{i, j+\frac{1}{2}}^{n+1}}^{n+\dot{m}_{k_{i, j+\frac{1}{2}}}^{n}}\right) \Delta X
$$

where $K_{\text {grid }}$ is the grid loss coefficient given as $K_{\text {grid }}=\frac{\zeta\left|v_{i, j+\frac{1}{2}}^{n}\right|}{2 \Delta X}$.

Eqs. (7), (8), and (9) express the final form of the fully implicit discretization of the interfacial shear, wall shear, and grid loss. It is comprised of two terms: one evaluated at the old timestep that is not captured by the original CTF linearization, and another term evaluated at the new timestep, which is two times higher than the CTF partially implicit discretization. The same linearization for the interfacial shear model with the drift flux approach was also developed and implemented into the code. The only task remaining is to proceed with testing and validation of the model, as summarized in Table 2. 
Table 2. Summary of fully implicit discretization derivation, implementation, and testing Momentum Equation

\begin{tabular}{rrrrrr}
\hline & & & \multicolumn{3}{c}{ Implicit discretization status } \\
\cline { 4 - 6 } Momentum term & Current status & End goal & Derivation & Implementation & Testing \\
\hline Advection flux & E & FI & No & No & No \\
Interfacial shear & PI & FI & Yes & Yes & No \\
Wall shear & PI & FI & Yes & Yes & No \\
Grid form loss & PI & FI & Yes & Yes & No \\
Mixing and void drift & E & FI & No & No & No \\
\hline
\end{tabular}

PI: partially implicit; E: explicit; FI: fully implicit

\subsubsection{Mass and Energy Equations}

In CTF, the nonlinear system of mass and energy equations resulting from the fully implicit discretization are linearized based on the Newton approach. In this method, the equation terms are linearized using Taylor series expansion around the primitive variables. The resulting first-order partial analytical derivatives of the discretized terms are known as the Jacobian matrix, and the remaining derivatives represent source or sink terms. A proper evaluation of these terms is crucial for obtaining a stable numerical solution. Because of the importance of the source and sink terms, a review was performed on the current linearization. This review revealed the following issues:

1. In the current CTF discretization, the source term resulting from the linearization of the inertial term-which is reflected in the form of difference in mass and energy between the new and old timestep-was not considered: it was canceled because of the previous lack of an outer iteration loop. Since the outer iteration was re-implemented, as described in the previous milestone report Salko et al. [2020b], this term must be taken into account to preserve mass and hence improve convergence.

2. The spatial discretization of the advection or convective terms in both mass and energy equations uses transported properties from the previous timestep. To improve the solution stability, we propose to express the cell outflow with transported properties using the new timestep and inflow with transported properties from neighboring mesh cells evaluated at the old timestep.

3. The mass and energy transfer resulting from mixing and void drift are discretized explicitly. This must be improved to the fully implicit form.

4. The set of equations, in addition to the appropriate constitutive relationships, does not form a complete set of equations for the variables at all node positions. These difference equations must be supplemented by additional relationships among variables at the edges and centers of mesh cells using a weighted donor cell approach that can help damp out flow oscillations to stabilize the solution. This is preferred over the current approach based on central differencing, which is less stable. An example of this issue is void fraction and density used in the evaluation of interfacial shear or wall shear. Any source term that is a function of the dependent variables must be discretized in upwind form of the dependent variables.

Summary of the improvement on linearization tasks are depicted in Table 3. In this study we are only 
concerned with improvements for the first and second issues. The third and fourth issues will be part of future work.

Table 3. Summary of fully implicit discretization derivation, implementation, and testing of mass and energy Equations.

\begin{tabular}{rrrrrrr}
\hline & & & \multicolumn{3}{c}{ Implicit discretization status } \\
\cline { 4 - 6 } Mass/energy term & Current status & End goal & Derivation & Implementation & Testing \\
\hline Advection flux & PI & FI & Yes & Yes & No \\
Inertial Term & FI & FI & Yes & Yes & Yes \\
Evaporation term & FI & FI & Yes & Yes & Yes \\
Wall heat & PI & FI & No & No & No \\
Mixing and void drift & E & FI & No & No & No \\
\hline
\end{tabular}

PI: partially implicit; E: explicit; FI: fully implicit

\subsubsection{Inertial Term}

The inertial term for both the energy and mass equations is given in the following form:

$$
\left(\left(\alpha_{k} \phi_{k}\right)_{i, j}^{n+1, m+1}-\left(\alpha_{k} \phi_{k}\right)_{i, j}^{n+1, m}\right) \frac{V_{i, j}}{\Delta t}
$$

where $(i, j)$ are the cell indices, $k$ is the phase index, subscript $n$ indicates the timestep, $m$ is the outer iteration index (as described in Salko et al. [2020b]), and $\phi_{k}$ is the transported properties defined as

$$
\phi_{k}= \begin{cases}\rho_{k} & \text { for mass equation } \\ \rho_{k} h_{k} & \text { for energy equation. }\end{cases}
$$

The linearization of the transported thermal property $\left(\alpha_{k} \phi\right)$ around the primitive variables can be obtained using Taylor series expansion:

$$
\begin{array}{r}
\left(\alpha_{k} \phi_{k}\right)_{i, j}^{n+1, m+1}=\left(\alpha_{k} \phi_{k}\right)_{i, j}^{n+1, m}+\left(\frac{\partial\left(\alpha_{k} \rho_{k}\right)}{\partial \alpha_{v}}\right)_{i, j}^{n+1, m} \Delta \alpha_{v_{i, j}}^{n+1, m+1} \\
+\left(\frac{\partial\left(\alpha_{k} \phi_{k}\right)}{\partial \alpha_{e}}\right)_{i, j}^{n+1, m} \Delta \alpha_{e_{i, j}}^{n+1, m+1}+\left(\frac{\partial\left(\alpha_{k} \phi_{v}\right)}{\partial \alpha_{v} h_{v}}\right)_{i, j}^{n+1, m} \Delta\left(\alpha_{v} h_{v}\right)_{i, j}^{n+1, m}+ \\
\left(\frac{\partial\left(\alpha_{k} \phi_{k}\right)}{\partial\left(1-\alpha_{v}\right) h_{\ell}}\right)_{i, j}^{n+1, m} \Delta\left(\left(1-\alpha_{v}\right) h_{\ell}\right)_{i, j}^{n+1, m}+\left(\frac{\partial\left(\alpha_{k} \phi_{k}\right)}{\partial P}\right)_{i, j}^{n+1, m} \Delta P_{i, j}^{n+1, m+1} \\
+\left(\frac{\partial\left(\alpha_{k} \phi_{k}\right)}{\partial P_{n c g}}\right)_{i, j}^{n+1, m} \Delta P_{n c g_{i, j}^{n+1, m+1}}^{n} .
\end{array}
$$


Note that $\Delta \alpha_{v}, \Delta \alpha_{e}, \Delta\left(\alpha_{v} h_{v}\right), \Delta\left(1-\alpha_{v}\right) h_{\ell}, \Delta P$, and $\Delta P_{n c g}$ represent the rate of change for which CTF is solving. The relationship between the system pressure and the gas partial pressure is established assuming that Dalton's law applies:

$$
P=P_{v}+P_{n c g}
$$

The derivative $\left(\frac{\partial\left(\alpha_{v} \rho_{v}\right)}{\partial P_{n c g}}\right)_{i, j}^{n+1, m}$ can be evaluated using the chain rule derivative:

$$
\left(\frac{\partial\left(\alpha_{v} \rho_{v}\right)}{\partial P_{n c g}}\right)_{i, j}^{n+1, m} \Delta P_{n c g_{i, j}^{n+1, m+1}}^{n+}=\left[\left(\frac{\partial\left(\alpha_{v} \rho_{v}\right)}{\partial P}\right)_{i, j}^{n+1, m} \times\left(\frac{\partial P}{\partial P_{n c g}}\right)_{i, j}^{n+1, m}\right] \Delta P_{n c g_{i, j}^{n+1, m+1}}^{n+}
$$

The derivative $\left(\frac{\partial P}{\partial P_{n c g}}\right)_{i, j}^{n+1, m}$ can be evaluated from Eq. (13) as $\left(\frac{\partial P}{\partial P_{n c g}}\right)_{i, j}^{n+1, m}=1$. Substituting this into Eq. (14) leads to the following:

$$
\left(\frac{\partial\left(\alpha_{v} \rho_{v}\right)}{\partial P_{n c g}}\right)_{i, j}^{n+1, m} \Delta P_{n c g_{i, j}^{n+1, m+1}}^{n+1}=\left(\frac{\partial\left(\alpha_{v} \rho_{v}\right)}{\partial P}\right)_{i, j}^{n+1, m} \Delta P_{n c g_{i, j}^{n+1, m+1}}
$$

Substituting Eq. (12) into Eq. (10) and rearranging terms in accordance with its proper rate of change lead to the contribution to the Jacobian (rjac) and source term (airs),

$$
\begin{aligned}
\operatorname{rac}_{i, 1} & =\frac{V_{i, j}}{\Delta t}\left(\frac{\partial\left(\alpha_{k} \phi_{k}\right)}{\partial P_{n c g}}\right)_{i, j}^{n+1, m} \\
\operatorname{rac}_{i, 2} & =\frac{V_{i, j}}{\Delta t}\left(\frac{\partial\left(\alpha_{k} \phi_{k}\right)}{\partial \alpha_{v}}\right)_{i, j}^{n+1, m} \\
\operatorname{rjac}_{i, 3} & =\frac{V_{i, j}}{\Delta t}\left(\frac{\partial\left(\alpha_{k} \phi_{k}\right)}{\partial\left(\alpha_{v} h_{v}\right)}\right)_{i, j}^{n+1, m} \\
\operatorname{rjac}_{i, 4} & =\frac{V_{i, j}}{\Delta t}\left(\frac{\partial\left(\alpha_{k} \phi_{k}\right)}{\partial\left(1-\alpha_{v}\right) h_{\ell}}\right)_{i, j}^{n+1, m} \\
\operatorname{rjac}_{i, 5} & =\frac{V_{i, j}}{\Delta t}\left(\frac{\partial\left(\alpha_{k} \phi_{k}\right)}{\partial \alpha_{e}}\right)_{i, j}^{n+1, m} \\
\operatorname{rjac}_{i, 6} & =\frac{V_{i, j}}{\Delta t}\left(\frac{\partial\left(\alpha_{k} \phi_{k}\right)}{\partial P}\right)_{i, j}^{n+1, m} \\
\operatorname{airs}_{i} & =\frac{V_{i, j}}{\Delta t}\left(\left(\alpha_{k} \phi_{k}\right)_{i, j}^{n+1, m}-\left(\alpha_{k} \phi_{k}\right)_{i, j}^{n, m}\right),
\end{aligned}
$$

where the first subscript $i$ of rjac and air represents the contribution of mass and energy equations, where 1 designates noncondensable gas mass, 2 is for the liquid mass equation, 3 is for the vapor energy, 4 is for the total liquid energy, 5 contributes to the droplet mass equation, and 6 is for the vapor mass equation. The second subscript designates the rate of the change of the dependent variables being solved for, which 
include: 1 , for $\Delta P_{n c g_{i, j}}^{n+1, m+1} ; 2$, for $\Delta \alpha_{v_{i, j}}^{n+1, m+1} ; 3$, for $\Delta\left(\alpha_{v} h_{v}\right)_{i, j}^{n+1, m+1} ; 4$, for $\Delta\left(\left(1-\alpha_{v}\right) h_{\ell}\right)_{i, j}^{n+1, m} ; 5$, which corresponds to $\Delta \alpha_{e_{i, j}}^{n+1, m+1}$; and 6 , for $\Delta P_{i, j}^{n+1, m+1}$. The partial derivatives are evaluated analytically, and the

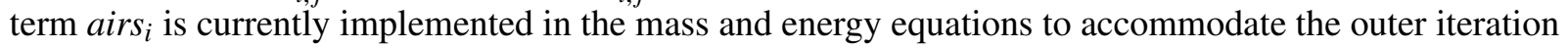
improvement. This modification is currently under testing.

\subsubsection{Linearization Improvements to Advection Terms}

This report shows only the discretization of flux out of the interface delimited by cell $j$ and $j+1$.

Linearization of other interfaces can be derived in the same manner. As stated above, the convective term in CTF is discretized using the donor cell principle associated with transported properties evaluated at the previous timestep. Thus, the convective term out of the cell can be given in the following form:

$$
\frac{\dot{m}_{k,(i, j+1 / 2)}^{n+1, m}}{\left(\overline{\left.\alpha_{k} \rho_{k}\right)_{i, j+1 / 2}^{n+1, m}}\right.}\left\{\begin{array}{ll}
\left(\alpha_{k} \phi_{k}\right)_{i, j}^{n+1, m} & \text { if } \dot{m}_{k,(i, j+1 / 2)}^{n+1, m} \geq 0 \\
\left(\alpha_{k} \phi_{k}\right)_{i, j+1}^{n+1, m} & \text { if } \dot{m}_{k,(i, j+1 / 2)}^{n+1, m}<0
\end{array},\right.
$$

where $\dot{m}_{k,(i, j+1 / 2)}^{n+1, m}$ is the phasic mass flow rate calculated at the interface $(i, j+1 / 2)$, and $\left({\overline{\alpha_{k} \rho_{k}}}_{i, j+1 / 2}^{n+1, m}\right.$ is an average of the thermal properties, evaluated as

$$
\left(\bar{\alpha}_{k} \rho_{k}\right)_{i, j+1 / 2}^{n+1, m}=0.5 \times\left(\left(\alpha_{k} \rho_{k}\right)_{i, j}^{n+1, m}+\left(\alpha_{k} \rho_{k}\right)_{i, j+1}^{n+1, m}\right) .
$$

The mass flow rate for phase $k$ can be written as a linear function of the pressure difference across the interface of interest:

$$
\dot{m}_{k,(i, j+1 / 2)}^{n+1, m}=\dot{m}_{k,(i, j+1 / 2)}^{n, m}+\left(\frac{\partial \dot{m}_{k}}{\partial \Delta P}\right)_{i, j}^{n+1, m}\left(\Delta P_{i, j}^{n+1, m+1}-\Delta P_{i, j+1}^{n+1, m+1}\right) .
$$

A donor cell for the transported cell properties is defined as

$$
\begin{gathered}
\left(\alpha_{k} \phi_{v}\right)_{i, j+1 / 2}^{D}= \begin{cases}\left(\alpha_{k} \phi_{k}\right)_{i, j}^{n+1, m} & \text { if } \dot{m}_{k,(i, j+1 / 2)}^{n+1, m} \geq 0 \\
\left(\alpha_{k} \rho_{k}\right)_{i, j+1}^{n+1, m} & \text { if } \dot{m}_{k,(i, j+1 / 2)}^{n+1, m}<0\end{cases} \\
D_{i, j+1 / 2}^{v}= \begin{cases}1 & \text { if } \dot{m}_{k,(i, j+1 / 2)}^{n+1, m} \geq 0 \\
0 & \text { if } \dot{m}_{k, i, j+1 / 2)}^{n+1, m}<0\end{cases}
\end{gathered}
$$

Substituting Eq. (19) into Eq. (17) leads to the Jacobian coefficients and source term: 


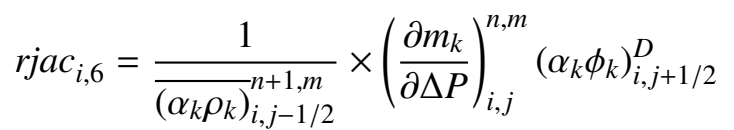

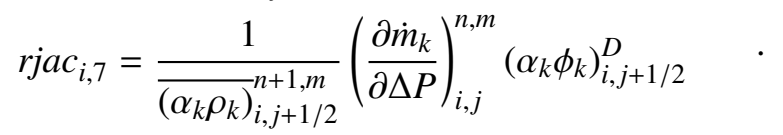

$$
\begin{aligned}
& \operatorname{airs}_{i}=\frac{\dot{m}_{v,(i, j+1 / 2)}^{n, m}}{\left(\alpha_{k} \rho_{v}\right)_{i, j+1 / 2}^{n+1, m}} \times\left(\alpha_{k} \phi_{k}\right)_{i, j+1 / 2}^{D}
\end{aligned}
$$

Eq. (21) is currently implemented in CTF. As the equation shows, the linearization involves only terms that originate from linearization of the velocity with respect to pressure at the interface. The contribution of the linearization of transported properties is not included due to the use of the explicit discretization. To improve on this, the team proposed to express the transported properties in fully implicit form. In this linearization, it is customary to express (1) flux out of the mesh cell as a function of the transported properties evaluated at the new timestep and (2) flux into the cell obtained with neighboring properties evaluated at the last timestep. The objective is to use new time information for the portion of the flux associated with the same cell as the equation at cell $(i, j)$. This makes the local solution more sensitive to variation in phase-change rates for cases in which flux can be predominately attributed to phase change in the cell for which the continuity equation is performed. Thus, we have

$$
\frac{\dot{m}_{k,(i, j+1 / 2)}^{n+1, m}}{\left(\overline{\alpha_{k} \rho_{k}}\right)_{i, j+1 / 2}^{n+1, m}} \begin{cases}\left(\alpha_{k} \phi_{k}\right)_{i, j}^{n+1, m+1} & \text { if } \dot{m}_{k,(i, j+1 / 2)}^{n+1, m} \geq 0 \\ \left(\alpha_{k} \phi_{k}\right)_{i, j+1}^{n+1, m} & \text { if } \dot{m}_{k,(i, j+1 / 2)}^{n+1, m}<0\end{cases}
$$

where the term $\left(\alpha_{k} \phi_{k}\right)_{i, j}^{n+1, m+1}$ represents the transported properties at the new time level (fully implicit). The new fully implicit time level is expressed by Eq. (12) in linearized form around the independent variables. The $\dot{m}_{k, i, j+1 / 2)}^{n+1, m}$ defines the mass flow, which is written as a linear function of the pressure difference across the interface. This is shown in Eq. (19). Then, replacing those terms with their expression, employing the definition of the donor cell property expressed as given by Eq. (20), and neglecting higher-order derivatives (Eq. (22), leads to the following: 


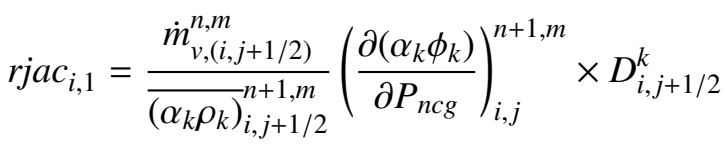

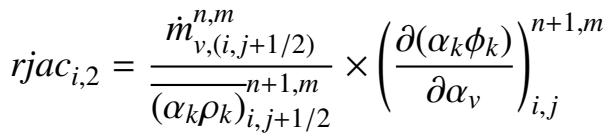

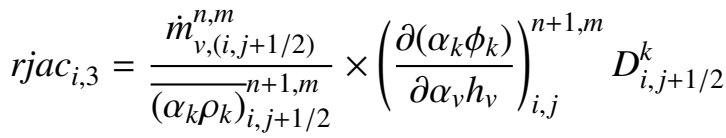

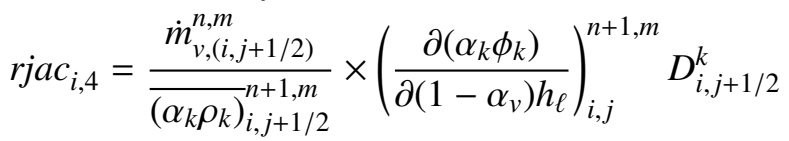

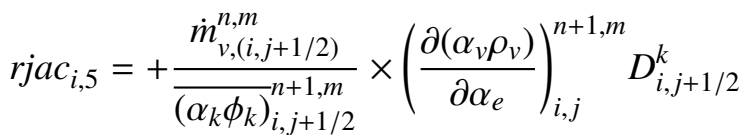

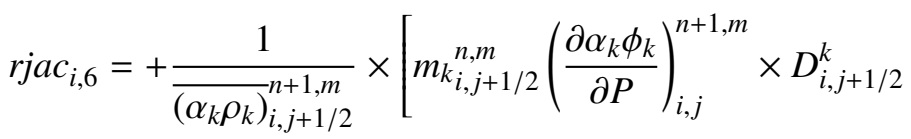

$$
\begin{aligned}
& \left.+\left(\frac{\partial m_{k}}{\partial \Delta P}\right)_{i, j}^{n, m}\left(\alpha_{k} \rho_{k}\right)_{i, j+1 / 2}^{D}\right]
\end{aligned}
$$

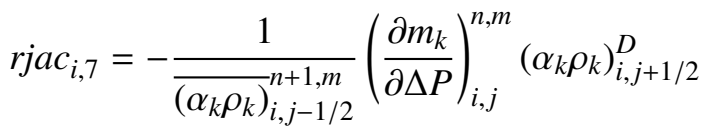

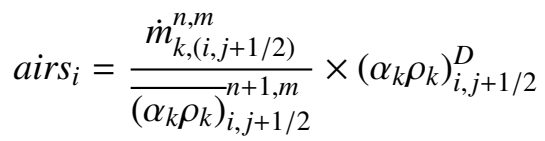

Eq. (23) is the final form of the improved linearization of the convective term for any interface and phase $k$ if the derivatives are evaluated analytically or numerically. This equation can degenerate to CTF's original linearization expressed by Eq. (21) if the donor cell properties $D_{i, j+1 / 2}^{k}$ are set to zero. This enhancement is currently being tested. The preliminary results indicate that the number of iterations is more or less the same as that of the CTF original implementation. This is expected: this correction has not been blended with the other correction that replaces the explicit discretization with the fully implicit one.

\subsection{REVIEW OF TIME STEPPING LOGIC}

Because of the semi-implicit linearization of the mass and energy equations, the CTF transient solution is CFL-limited. In practice, the purpose of this limitation is to prevent the pressure wave from "jumping" over a mesh cell in one given timestep, which can lead to numerical instability. The CFL limit will set the timestep size as shown in Eq. (24), where $\Delta t$ is the timestep size, $C$ is the nondimensional CFL number, $\Delta x$ is the length of the scalar mass and energy mesh cell (simply called "mesh cell" moving forward) under consideration, and $v$ is the fluid velocity in the mesh cell:

$$
\Delta t \leq C \frac{\Delta x}{v}
$$


A CFL number of 1.0 means that the fluid would traverse a single axial level in a single timestep, but a default value of 0.8 is used in CTF to further limit timestep size and to improve stability. Note that while CTF is CFL limited, this limiter will not necessarily cover all physics processes being modeled, some of which may require a finer time discretization (e.g., conduction heat transfer, phase change, etc.), which is why a CFL value of less than 1.0 is typically used. Because the fluid velocity and mesh cell length will be different in all cells, and because there are three fields to consider, CTF will find the minimum $\Delta x / v$ in the mesh to set the timestep size. However, CTF does not explicitly consider mesh cell velocity. Rather, it finds the total advection mass flow rate of each field out of the faces (both axial and lateral faces) of the mesh cell, converts that mass to a volumetric flux by dividing by $\alpha \rho$ of the scalar mass and energy cell for the field under consideration, and then divides this by the cell volume to get a value that has equivalent units to $\Delta x / v$. This process is implemented for each field separately, and the minimum of each $\Delta x / v$ term is then used to determine the limiting value for that cell. This is repeated for every mass and energy cell in the mesh to find the minimum value for the entire mesh. This value is then used in Eq. (24) to obtain the timestep size for the next iteration.

Because the density of the scalar cell is being used instead of the momentum cell where the mass flow rate is defined, the result is not truly the velocity in the momentum cell. Furthermore, the length of the momentum cell is not explicitly considered in this process.

A more straightforward calculation of the CFL limit involves looping over all momentum cells in the model (both axial and lateral) and all fields in the solution (liquid, vapor (steam plus noncondensable gas mixture), and droplets), and calculating the minimum $\Delta x / v$ for each cell explicitly. The velocity shall be based on the momentum cell void and density, and $\Delta x$ is the momentum cell length. The minimum of all momentum mesh cells is used to calculate the timestep size. This approach was implemented and used in lieu of the original form to test the impact. For all cases tested, it was observed that the timestep size grows much larger than with the original approach, which subsequently leads to most tests becoming unstable and failing due to the current explicit linearization that is utilized. Fig. 1 shows the average increase in timestep size for five selected validation test categories. The timestep size increase was calculated by finding the maximum timestep size using the original CFL limit and the maximum timestep size using the new CFL limit. The difference was obtained for each test in the category, and the differences were averaged to obtain the results shown in Fig. 1.

Note that the $y$-axis was set to logarithmic because the PWR Subchannel and Bundle Tests (PSBT) bundle tests see a $10 \times$ increase in timestep size. All other tests resulted in roughly a $2 \times$ increase in timestep size. Upon further investigation, a defect was discovered in the original CFL approach. The vapor velocity used for the CFL limit was being calculated as follows:

$$
v_{\text {limit }}=\frac{\dot{m}_{v}}{\alpha_{v} \rho_{v} A}+\frac{\dot{m}_{g}}{\alpha_{v} \rho_{g} A}
$$

where $\dot{m}_{v}$ and $\dot{m}_{g}$ are vapor and noncondensable gases defined from the total steam (vapor + gas) flow rate $\dot{m}_{s}$ as

$$
\begin{aligned}
& \dot{m}_{v}=\frac{\dot{m}_{s} \rho_{v} \alpha_{v}}{\left(\alpha_{v} \rho_{v}+\alpha_{v} \rho_{n c g}\right)} . \\
& \dot{m}_{g}=\frac{\dot{m}_{s} \rho_{v} \alpha_{v}}{\left(\alpha_{v} \rho_{v}+\alpha_{v} \rho_{n c g}\right)} .
\end{aligned}
$$




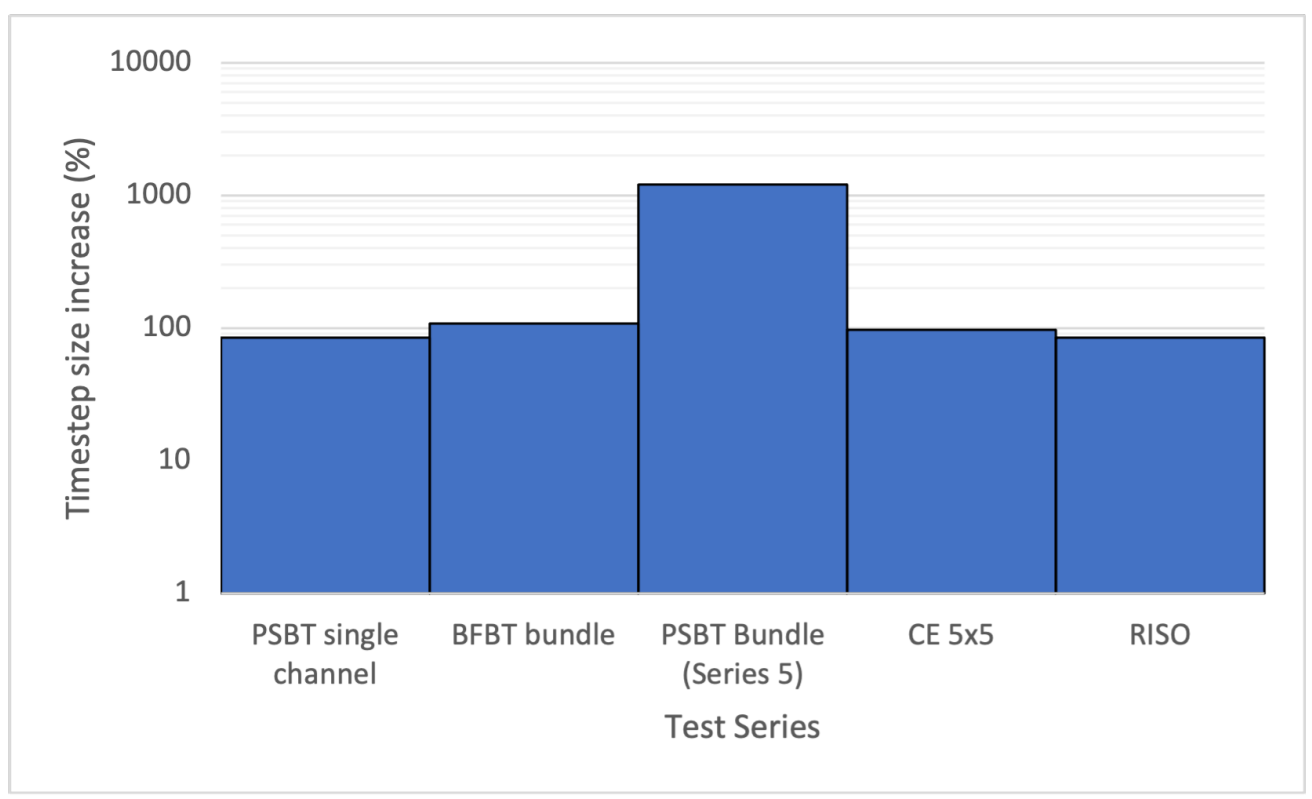

Figure 1. Average percentage increase in maximum timestep size in the solution for five selected validation test categories when switching from the original Courant-Friedrichs-Lewy approach to the new one.

In the equation, $v_{\text {limit }}$ is the vapor velocity out of the cell to be considered for determination of the timestep size. The $\dot{m}$ term is the mass flow rate, where $v$ represents the vapor and $g$ represents the noncondensable gas. The remaining terms represent volume fraction $(\alpha)$, density $(\rho)$, and cross sectional flow area of the channel $(A)$. Note that CTF assumes that the noncondensable gas and steam occupy the same volume, which is represented by $\alpha_{v}$. It is clear from these expressions that this will lead to the velocity of the vapor and the velocity of the gas-which should be identical—being added together. This leads to a velocity that is twice what it should be, and it explains why most tests result in a $2 \times$ increase in timestep size after the issue is corrected. The correct way to express this equation is as follows:

$$
v_{\text {limit }}=\frac{\dot{m}_{v}+\dot{m}_{g}}{\alpha_{v}\left(\rho_{v}+\rho_{g}\right) A} .
$$

In this case, the total mass flow rate of vapor and gas is divided by the total density of the mixture.

For the PSBT tests, the timestep size will still be several times larger than that obtained in the original approach, even after this issue is corrected. Further investigation revealed that the timestep discrepancy is caused by the highly nonuniform mesh used in the PSBT tests. The region of the mesh that causes this discrepancy is shown in Fig. 2.

As noted above, the original CFL approach uses the scalar mesh cell height to calculate the $\Delta x / v$ term, whereas the new approach uses the momentum cell height. As shown in the figure, the height of the first scalar cell is $2.5 \times 10^{-3} \mathrm{~m}$. Because of the mesh nonuniformity, the height of the momentum cell (shown in red) between the two scalar cells is $4.0 \times 10^{-2} \mathrm{~m}$, which is roughly an order of magnitude larger and explains why the timestep size will be an order of magnitude larger. By using the scalar cell height, the 


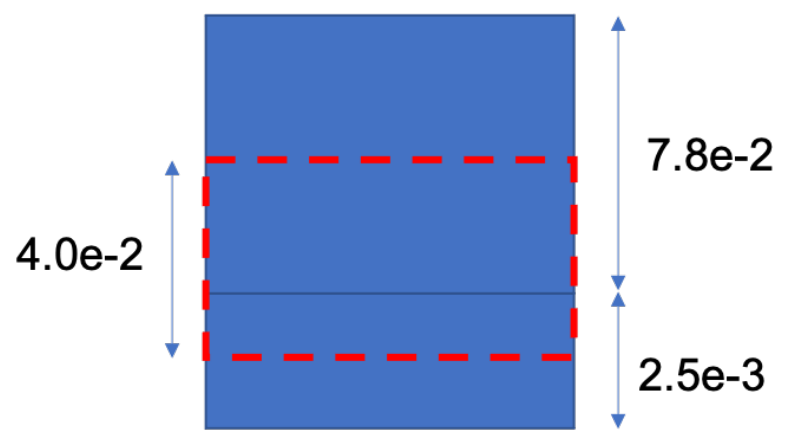
Figure 2. Nonuniform region of PSBT axial mesh (not to scale) that causes the original and new
Courant-Friedrichs-Lewy implementation to predict different timestep sizes.

timestep size can be significantly smaller than the required value. As noted previously, many more tests fail at the larger timestep because of numerical instability. This is because of the explicitness of the linearization as described in the previous sections. Therefore, the team suggested that improvements to the linearization should be implemented before the corrections noted in this section can be made and the new CFL approach can be implemented in the production version of CTF. This will be implemented as part of future work.

\subsection{PARALLELIZATION IMPROVEMENTS}

CTF uses Message Passing Interface (MPI) to parallelize the solution. MPI is a distributed-memory parallelization scheme, meaning that different processors solve different parts (domains) of the problem. The separate processors only store data relevant to the particular domain they are solving, meaning that boundary data must be shared throughout the solution to ensure the solution is consistent with a serial, nonparallel solution. The process for dividing the problem into smaller domains is performed by the preprocessor Xml2ctf. For BWR models, this was traditionally done by using an assembly as the basis for one domain. In other words, a model with 140 assemblies would require 140 processors to perform the solution.

For PWR models, Xml2ctf was set up to perform more refined domain decompositions than one processor per assembly, allowing the user to use as many as 16 processors per assembly. To improve performance of BWR models, it was decided that Xml2ctf should be modified to allow for using more processors per assembly. One hurdle for this activity was solution of the pressure matrix. In PWR models, a single pressure matrix is solved for the entire system, which is necessary because all subchannels in the system are connected. However, for BWR models, the lower and upper plenums, where assembly channels connect, are not explicitly modeled, so significant runtime savings can be realized by solving only one pressure matrix per assembly. Furthermore, although an iterative matrix solver can be used for PWR models, the complexity of two-phase flow found in BWR models requires that a direct solver be used to reduce solution error. Even though iterative solvers scale nicely when moving from a single assembly model to full core, the SuperLU solver experiences significant performance issues when scaled up to full core. Therefore, it was necessary to continue solving one pressure matrix per assembly, even when multiple processors are used to solve that assembly. 
To address this issue, a global indexing system was implemented on an assembly basis. In other words, each subchannel in the assembly receives a unique index that distinguishes it from all other subchannels in the assembly. Furthermore, an MPI subcommunicator was created for each assembly, which is then passed to Portable, Extensible Toolkit for Scientific Computation (PETSc) when setting up the matrix solver. The global subchannel indices are then used to set and obtain the pressure matrix coefficient and solution data when communicating with PETSc.

The other issue addressed for this activity was modification of Xml2ctf to perform the domain decomposition. For the initial implementation, only four processors per assembly were targeted as the most refined decomposition. The algorithm used to decompose the problem attempts to divide the problem into four equally sized squares. For a model with an odd number of subchannels, some of the squares will be bigger than others. For example, the domain decomposition is shown for a GE-14 assembly geometry in Fig. 3. The domain owner is denoted by color in the figure. Because there are 11 subchannels in a row or column of the assembly map, some domains are smaller than others; however, the number of pins in each domain is more balanced because they can be evenly divided.

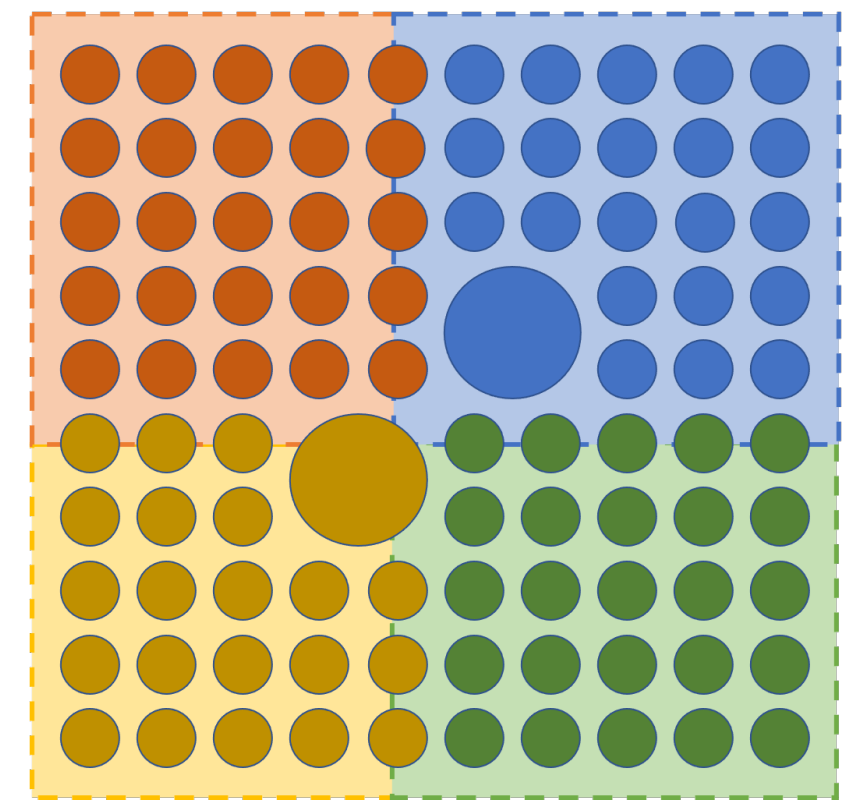

Figure 3. Domain decomposition of a GE-14 assembly with different MPI domains denoted by color.

A performance study was conducted for the single assembly case shown in Fig. 3. The case was run with one processor per assembly and four processors per assembly (using the domain decomposition shown in the figure). The results of the performance improvement for various parts of the CTF solution algorithm are shown in Table 4. The data can also be visualized in a pie chart as shown in Fig. 4; this illustrates the breakdown of time spent in sections of the code and shows how performance improved. Overall, a $2.4 \times$ speedup was observed when using four processors, resulting in a parallel efficiency of about $60 \%$. The table shows that the momentum equation solution and pressure matrix solution are the two most poorly scaling parts of the code. The momentum equation solution contains the majority of the MPI data transfers, so its poor scaling is expected. The pressure matrix solve section is entirely performed by SuperLU, so more investigation into the solver options may be needed to improve the efficiency of that section of the code. 
Table 4. Summary of performance improvement when solving a single GE-14 assembly using four processors vs. one processor

\begin{tabular}{rlll}
\hline Algorithm section & 1 processor & 4 processors & Speedup \\
\hline Heat transfer & 67 & 20 & 3.4 \\
Conduction solve & 114 & 31 & 3.7 \\
Momentum solve & 120 & 65 & 1.8 \\
Mass and energy solve & 26 & 8 & 3.4 \\
Pressure matrix & 106 & 53 & 2.0 \\
Variable update & 22 & 9 & 2.5 \\
Total & 438 & 194 & 2.4 \\
\hline
\end{tabular}

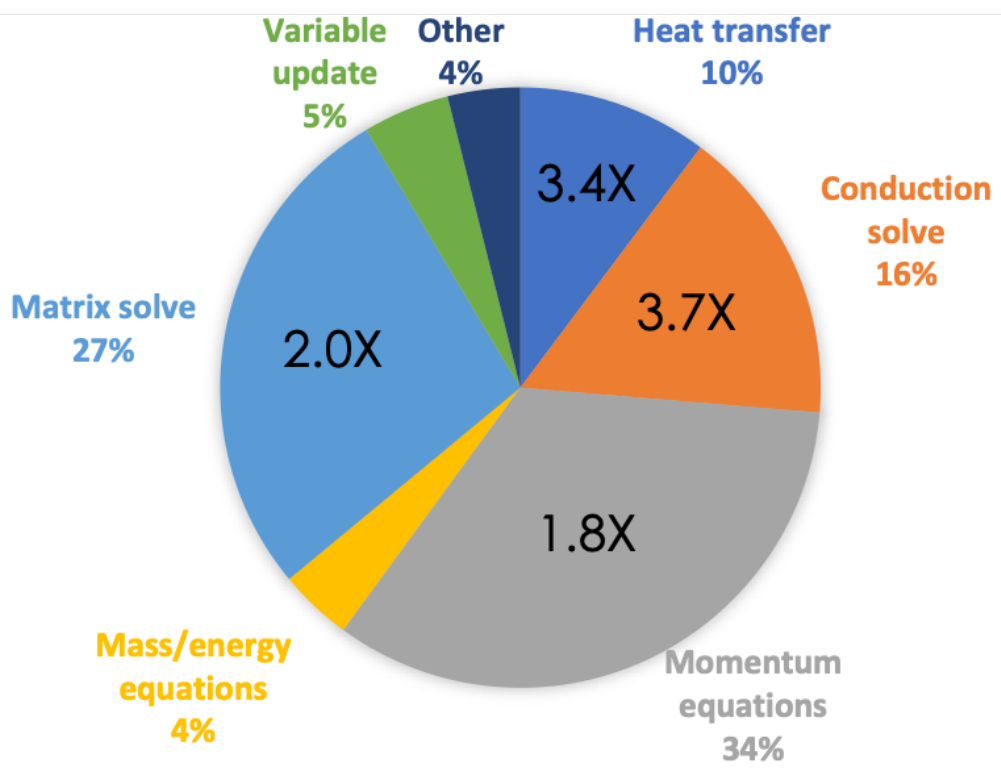

Figure 4. Performance improvement of various sections of CTF when increasing the number of processors from 1 to 4 for the GE-14 single-assembly case. Percentage of time spent in each code section (for the 4-processor case) is shown below the section heading. 
To investigate the performance improvements for a larger problem, a quarter symmetry model of Peach Bottom Unit 2 was run on the Idaho National Laboratory (INL) Sawtooth cluster. Results are shown in Table 5. The performance improvement for most code sections is consistent with the single assembly case, except for the pressure matrix solve, the scaling of which further degrades. This supports the finding that the SuperLU solver options should be further investigated in the future to improve code performance. The independent variable update performance also degraded considerably over the single assembly case, but it makes up a much smaller portion of the total CTF runtime. Furthermore, as in the momentum equation solution section, a number of MPI operations are performed, accounting for its poor scaling. Additional gains may also be made by improving the balancing of domain sizes between processors over the simple approach that was taken here; however, this should follow improving the scaling of the pressure matrix solve in terms of priority. If the scaling is improved, it will also be possible to use more than four processors per assembly and further reduction in runtime.

Table 5. Summary of performance improvement when solving a quarter-symmetry Peach Bottom Unit 2 model using 4 processors vs. 1 processor

\begin{tabular}{rlll}
\hline Algorithm Section & 1 processor & 4 processors & Speedup \\
\hline Heat transfer & 490 & 131 & 3.7 \\
Conduction solve & 746 & 199 & 3.7 \\
Momentum solve & 944 & 597 & 1.6 \\
Mass and energy solve & 176 & 55 & 3.2 \\
Pressure matrix & 957 & 668 & 1.4 \\
Variable update & 192 & 113 & 1.7 \\
Total & 3629 & 1813 & 2.0 \\
\hline
\end{tabular}

\section{GEOMETRY MODELING IMPROVEMENTS}

\subsection{NONUNIFORM CORES}

The nonuniform cores feature allows a user to mix different fuel assembly types (having a different number of pins in the lattice) in the same core model for BWR models. There were two obstacles to setting up cores with assemblies containing different fuel lattices. First, the format for specifying rod/subchannel core maps was not flexible enough to allow different assembly designs to be specified. Second, the HDF5 writer did not know how to format the data, which stems from the first issue of not having complete map data. To address these problems, a new format for entering rod/subchannel map data was developed for the CTF input deck. An example of the input format is shown in Fig. 5.

The assembly map information is provided first by supplying the core map dimensions and symmetry option, followed by the two-dimensional (2D) map of assembly indices. After this, the \{assembly_id_maps\} section is entered, which provides the 2D rod and channel index maps for each assembly. The rod and channel indices are unique and global to the entire model. The case denoted by this example is a symmetry case, which is why only three assembly indices are provided (one per each solved assembly). Assemblies that appear on the other side of the symmetry line shall not be entered. This new format provides the flexibility for the user to enter a map of assemblies of different sizes in the core model. 


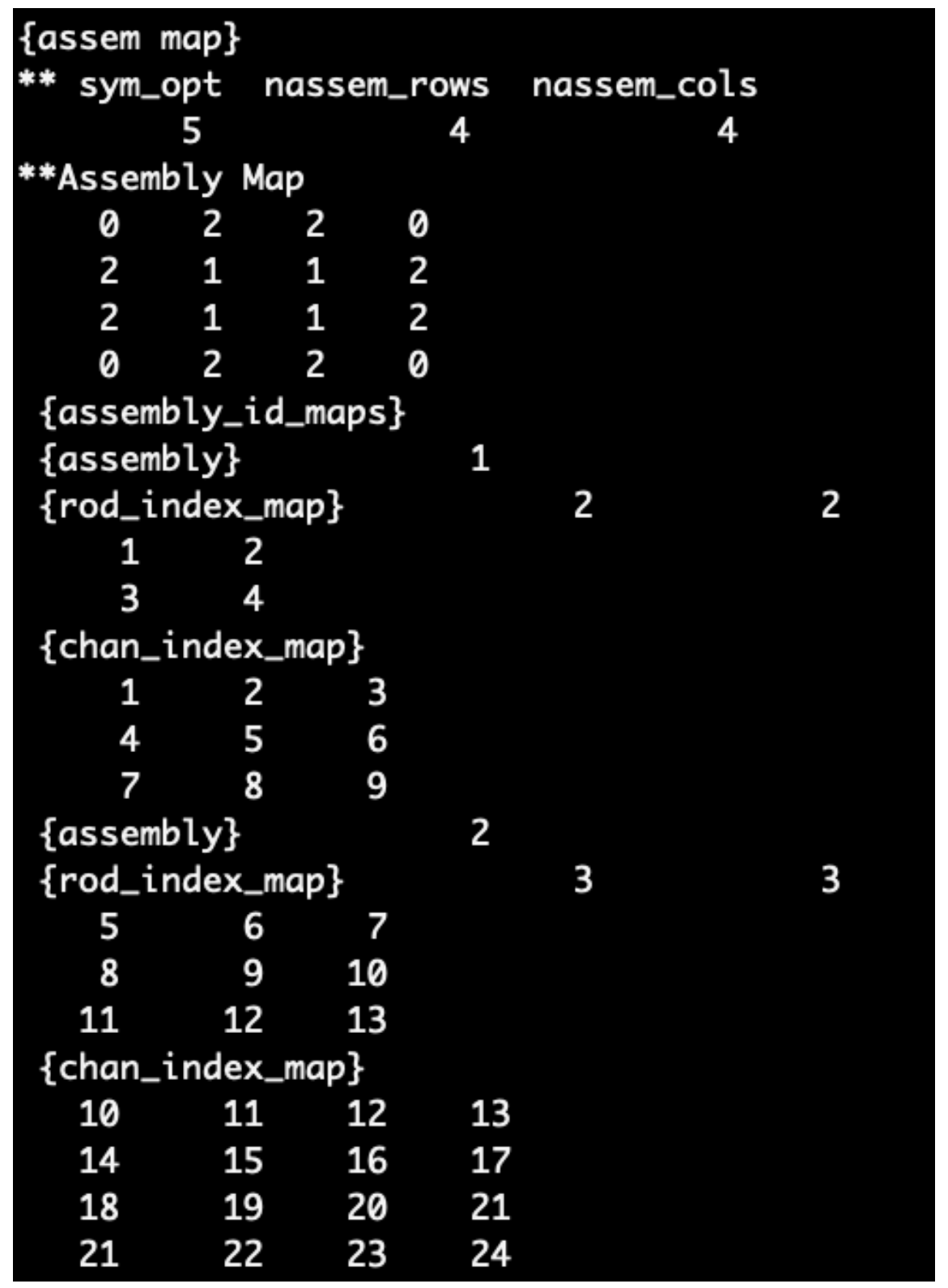

Figure 5. Example of the new model map input format using BWRs in the CTF input deck. 
This input format is only used for BWR models, which is identified in Card 1 of the CTF input deck. Note that this new input format will be automatically generated from the VERAIn file when a BWR model is being generated. In CTF, The assumption is made that the channel map will always be one row/column larger than the associated pin map. If any blank spots appear in the channel map (e.g., because of large water rods), they shall be denoted with a zero.

In addition to implementing the new input format, a refactor of the model map classes was required to store and use this new map data in CTF. Because PWR models still use the old map format, the RodMap and ChanMap classes were converted to abstract classes, with extensions for BWRRodMap, BWRChanMap, PWRRodMap, and PWRChanMap. Internally, the correlation class data were made private, and the interface to the new procedures was formally defined to ensure that no access of class data was being performed outside the class module. Deferred procedures were used for nearly all type-bound procedures to minimize disruptions to the rest of CTF. After the new input format and model map class design were implemented, it was possible to update the HDF5 writer to correctly format output data for BWR models with different assembly types. To test the feature, a mini-core problem containing 4 assemblies was created: 2 assemblies were $8 \times 8$ pins, and 2 were $10 \times 10$ pins. Fig. 6 shows the radial void distribution results for this problem as visualized by VERAView from the updated HDF5 file.

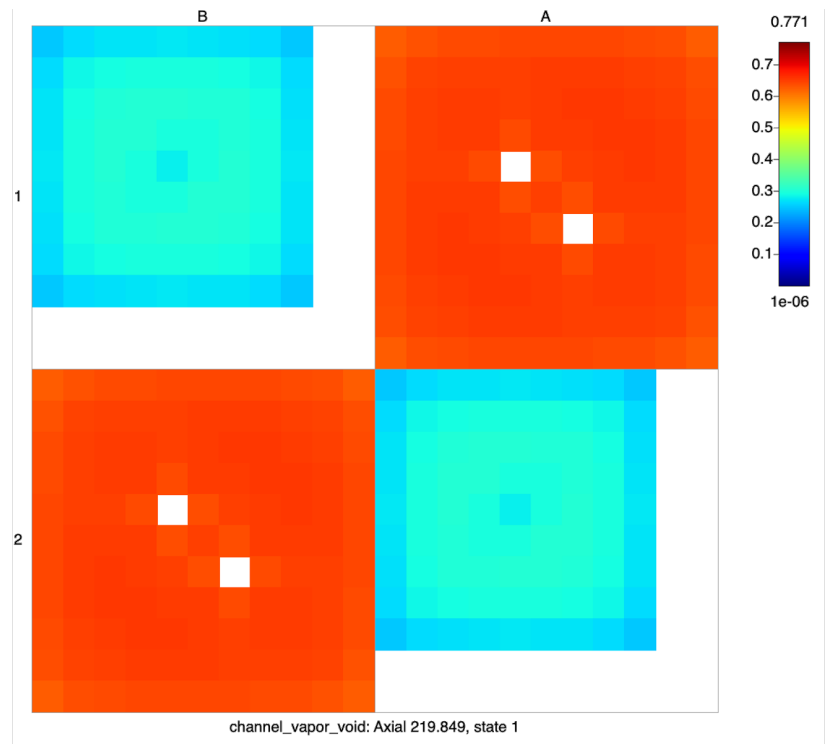
Figure 6. Outlet radial void distribution in a mini-core BWR model
which includes mixed assembly types $(8 \times 8$ and $10 \times 10)$.

Nominal BWR operating conditions and a uniform power distribution are used for the problem, but because the losses in the $10 \times 10$ geometry are different than those in the $8 \times 8$ geometry, more flow goes through the $8 \times 8$ assemblies, leading to a higher void in the $10 \times 10$ assemblies.

\subsection{UPPER PLENUM}

At this writing, the current BWR modeling practice using VERA is to model up to the end of heated length (EOHL) only; however, there may be $\mathrm{T} / \mathrm{H}$ behavior of interest to capture in the upper plenum region 
because the outlet quality will impact steam separator performance. It is already possible for CTF to model this region; however, it was not tested through VERA until the time of this report. Some modifications to MPACT were required to include this region in the model because the standard practice is to not model the region above the active region. An option was added to the VERAIO input that allows the user to model the lower plenum, upper plenum, both regions, or only the active region. A model of Peach Bottom Unit 3, Cycle 17 was developed that includes the upper plenum region of the core. The model was run in two ways: first, it was run with standalone CTF and the upper plenum region edits included, and second, it was run with coupled VERA with the upper plenum included in one case and not included in another so that a comparison could be made.

\subsubsection{CTF analysis}

To include the upper plenum effects in the CTF model and obtain results for this region, the axial_edit_bounds in VERAIO input was modified to include the roughly $30 \mathrm{~cm}$ high region between the EOHL and assembly outlet. Unfortunately, there were issues in the coupling between MPACT and CTF that prohibited performing a coupled solution when this region was included. This is still under investigation and will be resolved in a future release. Therefore, the task was completed by instead assessing a standalone CTF model of the core with the upper plenum included. To capture the power shape, a coupled VERA simulation of this core was run without the upper plenum region, and this file was read by $\mathrm{CTF}$ to set the power distribution in the active region (power was zero in the upper plenum region) and perform a standalone simulation.

The fuel assembly design for this core was GE-14, which includes two large water rods that have decreased diameters near the bottom and top of the core as well as one set of part-length rods. The axial varying water rod diameter decreases in the upper plenum region. As shown in Fig. 7, the flow areas around the large water rods increase from the EOHL toward the upper plenum. This geometric feature leads to quality and void increasing because the void migrates to the more open flow area surrounding the large water rods. Fig. 8 shows an axial cutaway of six selected assemblies in the core and the subchannel cross-flow area. Row 5 of the subchannels in the bundle lattice is shown, which runs through one of the large water rods and adjacent to several part-length rods. The figure indicates that subchannel flow areas open up at level 37 because of the part-length rods terminating. Additionally, the flow area is slightly more open in subchannels adjacent to the large water rods in levels 1 and 2 before the water rod diameter increases in level 3. Likewise, the flow area of the subchannels near the large water rods opens up again in level 56 as a result of the large water rod diameter decreasing.

Fig. 9 shows the difference in quality between adjacent levels in a selected assembly (Assembly N-18) to further highlight the trend. Fig. 9b shows that, in the heated region, quality decreased in the corner subchannels and the subchannels surrounding the water rods the areas of which are smaller than the interior subchannels. Quality increased in the interior subchannels, and it increased by a greater degree in the center subchannel, which has the most open flow area. This behavior is due to the void drift phenomenon. However, in the upper plenum, the trend reverses for the most part: quality decreased in the interior channels and increased in the channels around the large water rods because the flow area of these subchannels opened up (see Fig. 7b).

Finally, the mass flow rate-weighted, bundle-averaged equilibrium quality was calculated for the assembly at the last three axial levels, the last level being the upper plenum level. These values were 0.277, 0.279, 


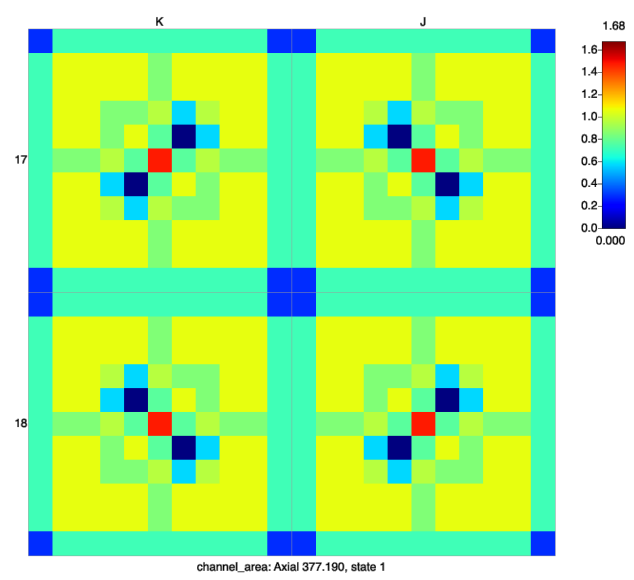

(a) Subchannel flow areas in last level of the core's heated region.

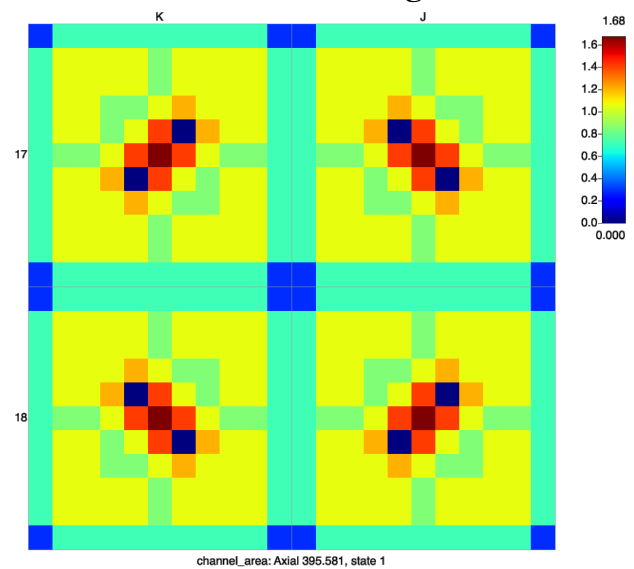

(b) Subchannel flow areas in the upper plenum region.

Figure 7. Subchannel cross sectional flow areas in the last heated level and upper plenum region of the core for four selected fuel assemblies. 


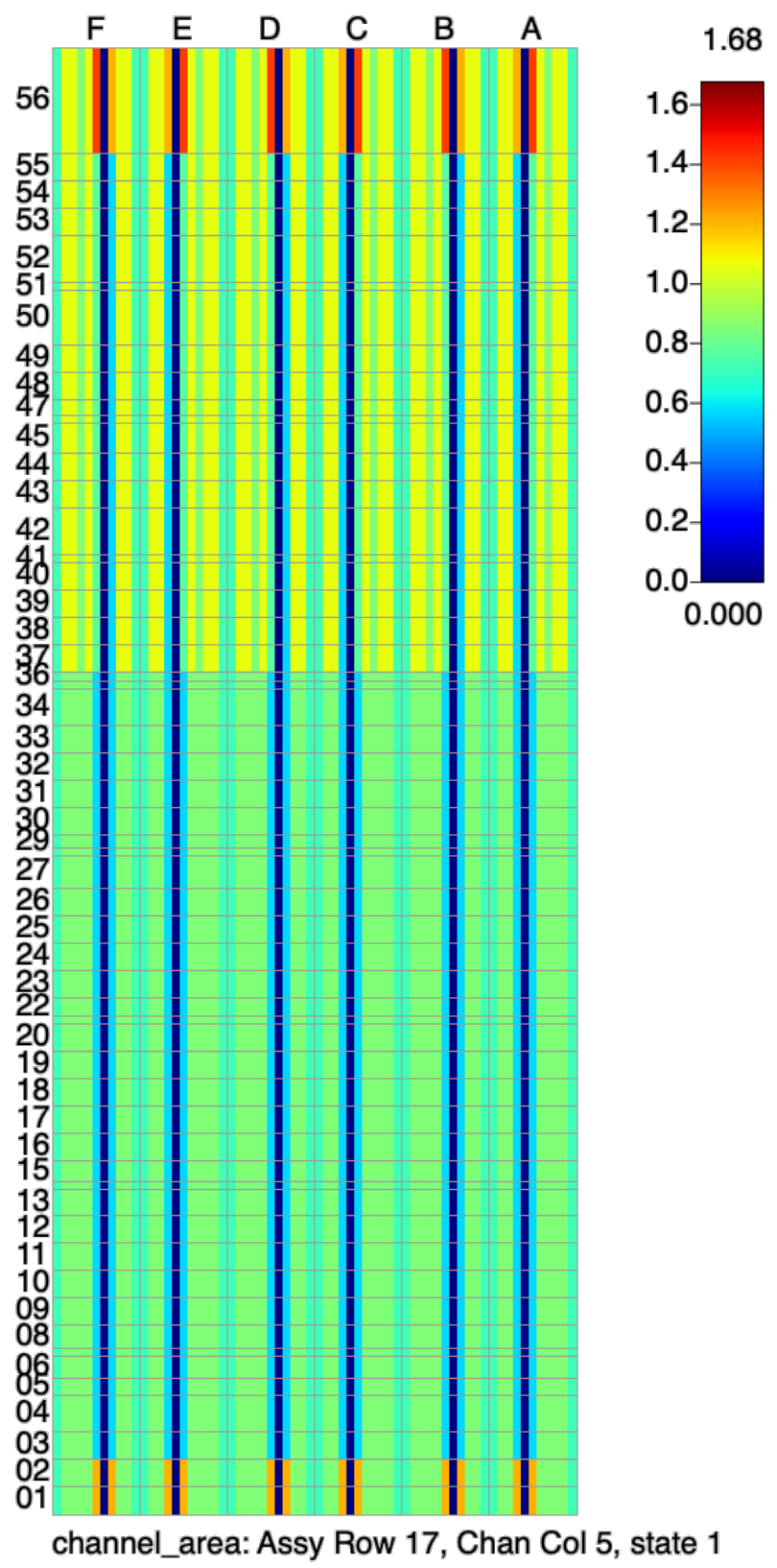

Figure 8. Axial cutaway of the core made through row 5 of subchannels in the assembly lattice. The cutaway is shown for six selected assemblies and denotes how the cross sectional area of the subchannels changes along the axial direction because of part-length rods and axial varying water rods. 


$\begin{array}{rrrrrrrrrrrr}0.007 & -0.009 & -0.007 & -0.006 & -0.006 & -0.007 & -0.006 & -0.005 & -0.006 & -0.008 & 0.008 \\ -0.009 & -0.022 & -0.018 & -0.017 & -0.018 & -0.003 & -0.017 & -0.016 & -0.017 & -0.021 & -0.008 \\ -0.007 & -0.018 & -0.013 & -0.013 & -0.014 & 0.005 & -0.005 & -0.007 & -0.011 & -0.017 & -0.006 \\ -0.006 & -0.017 & -0.013 & 0.003 & 0.006 & 0.033 & 0.077 & 0.036 & -0.007 & -0.016 & -0.005 \\ -0.006 & -0.018 & -0.014 & 0.006 & 0.001 & 0.062 & 0.000 & 0.077 & -0.004 & -0.016 & -0.005 \\ -0.007 & -0.003 & 0.005 & 0.033 & 0.062 & 0.038 & 0.062 & 0.033 & 0.006 & -0.003 & -0.006 \\ -0.006 & -0.017 & -0.005 & 0.077 & 0.000 & 0.062 & 0.002 & 0.007 & -0.013 & -0.018 & -0.006 \\ -0.005 & -0.016 & -0.007 & 0.036 & 0.077 & 0.033 & 0.007 & 0.004 & -0.012 & -0.016 & -0.005 \\ -0.006 & -0.017 & -0.011 & -0.007 & -0.004 & 0.006 & -0.013 & -0.012 & -0.013 & -0.017 & -0.006 \\ -0.008 & -0.021 & -0.017 & -0.016 & -0.016 & -0.003 & -0.018 & -0.016 & -0.017 & -0.021 & -0.008 \\ 0.008 & -0.008 & -0.006 & -0.005 & -0.005 & -0.006 & -0.006 & -0.005 & -0.006 & -0.008 & 0.008\end{array}$

(a) Quality distribution at the outlet of the upper plenum minus the quality distribution in the preceding level.

$\begin{array}{rrrrrrrrrrrr}-0.027 & -0.006 & -0.008 & -0.008 & -0.007 & -0.007 & -0.007 & -0.008 & -0.007 & -0.006 & -0.027 \\ -0.006 & 0.014 & 0.011 & 0.011 & 0.013 & -0.007 & 0.013 & 0.012 & 0.011 & 0.014 & -0.006 \\ -0.008 & 0.011 & 0.008 & 0.010 & 0.012 & -0.011 & 0.010 & 0.010 & 0.009 & 0.012 & -0.007 \\ -0.008 & 0.011 & 0.010 & -0.011 & -0.011 & -0.014 & -0.042 & -0.013 & 0.010 & 0.012 & -0.008 \\ -0.007 & 0.013 & 0.012 & -0.011 & 0.008 & -0.008 & 0.000 & -0.042 & 0.010 & 0.013 & -0.007 \\ -0.007 & -0.007 & -0.011 & -0.014 & -0.008 & 0.042 & -0.008 & -0.013 & -0.010 & -0.007 & -0.007 \\ -0.007 & 0.013 & 0.010 & -0.042 & 0.000 & -0.008 & 0.008 & -0.010 & 0.012 & 0.013 & -0.007 \\ -0.008 & 0.012 & 0.010 & -0.013 & -0.042 & -0.013 & -0.010 & -0.010 & 0.010 & 0.011 & -0.008 \\ -0.007 & 0.011 & 0.009 & 0.010 & 0.010 & -0.010 & 0.012 & 0.010 & 0.008 & 0.011 & -0.008 \\ -0.006 & 0.014 & 0.012 & 0.012 & 0.013 & -0.007 & 0.013 & 0.011 & 0.011 & 0.014 & -0.006 \\ -0.027 & -0.006 & -0.007 & -0.008 & -0.007 & -0.007 & -0.007 & -0.008 & -0.008 & -0.006 & -0.027\end{array}$

(b) Quality distribution at the end of the heated length minus the quality distribution in the preceding level.

Figure 9. Quality distribution compared between adjacent levels. 


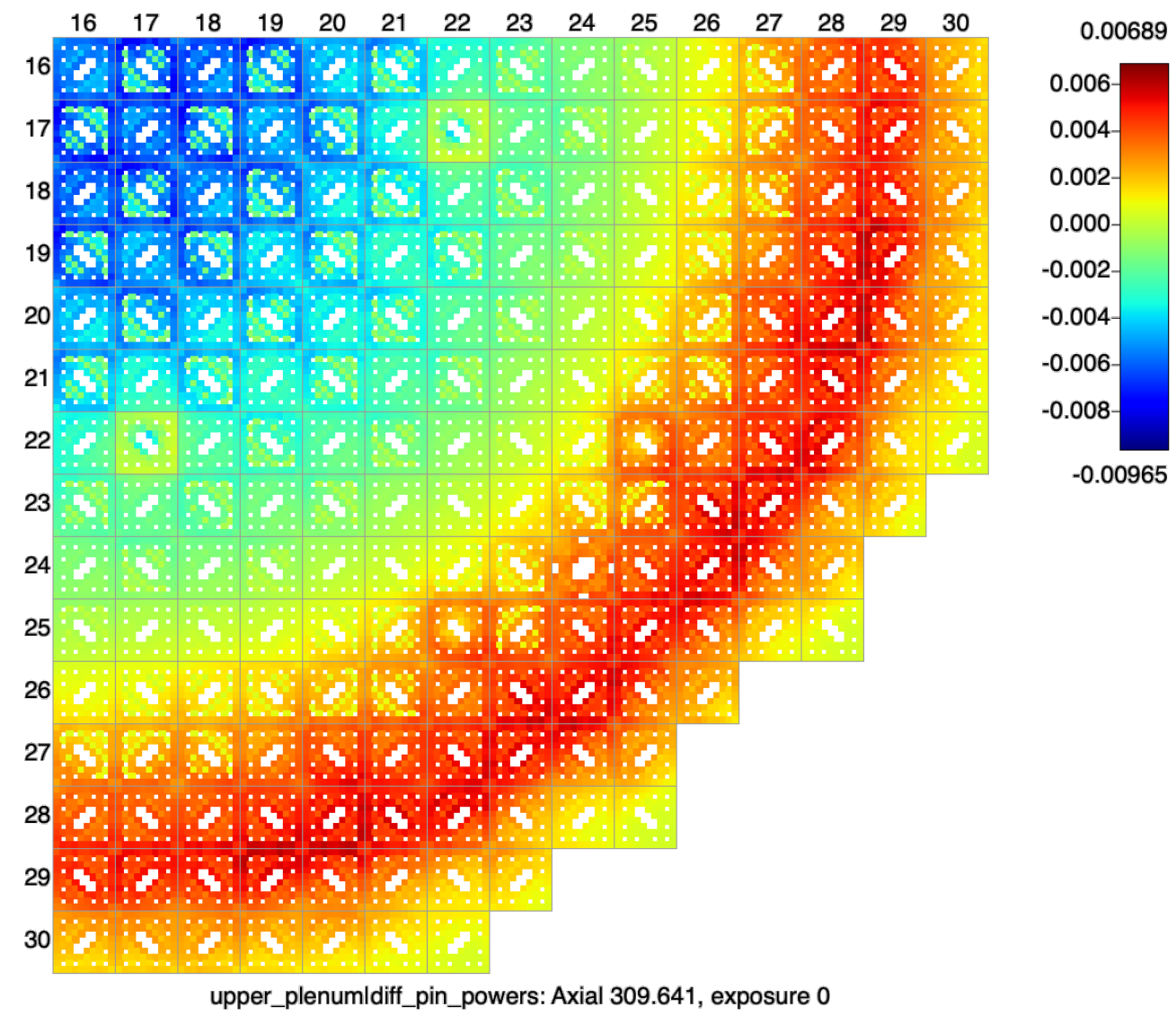

\section{Figure 10. The difference in power distribution between a model of Peach Bottom with the upper plenum compared to a model without the upper plenum.}

and 0.277 , respectively. The quality increased in the last heated level because of the relatively small power input in that region, as expected. There is a slight drop in quality in the upper plenum region. Note that there was no heat transfer through the channel box wall (the wall is assumed to be adiabatic), so this drop was unexpected. However, recall that CTF performs a pseudotransient to march to steady state, and this small discrepancy from the lower level is caused by the steady state tolerance.

\subsubsection{VERA analysis}

For the coupled analysis, one case was run with no modeling of the lower or upper plenum regions; only the active region was modeled. A second case was run with the upper plenum region modeled. Currently, writing edits for the upper plenum region in a coupled VERA simulation is not supported, so only the active region results are written to the results file. The effect on the power distribution was found to be relatively small; the largest difference in the pin power factor was 0.01 . A core cross section plot of the differences (upper plenum case minus no upper plenum case) is shown in Fig. 10. The largest differences were observed in the mid-to-upper core region, and power was higher in the outer ring and lower in the center when the upper plenum was included in the model. 


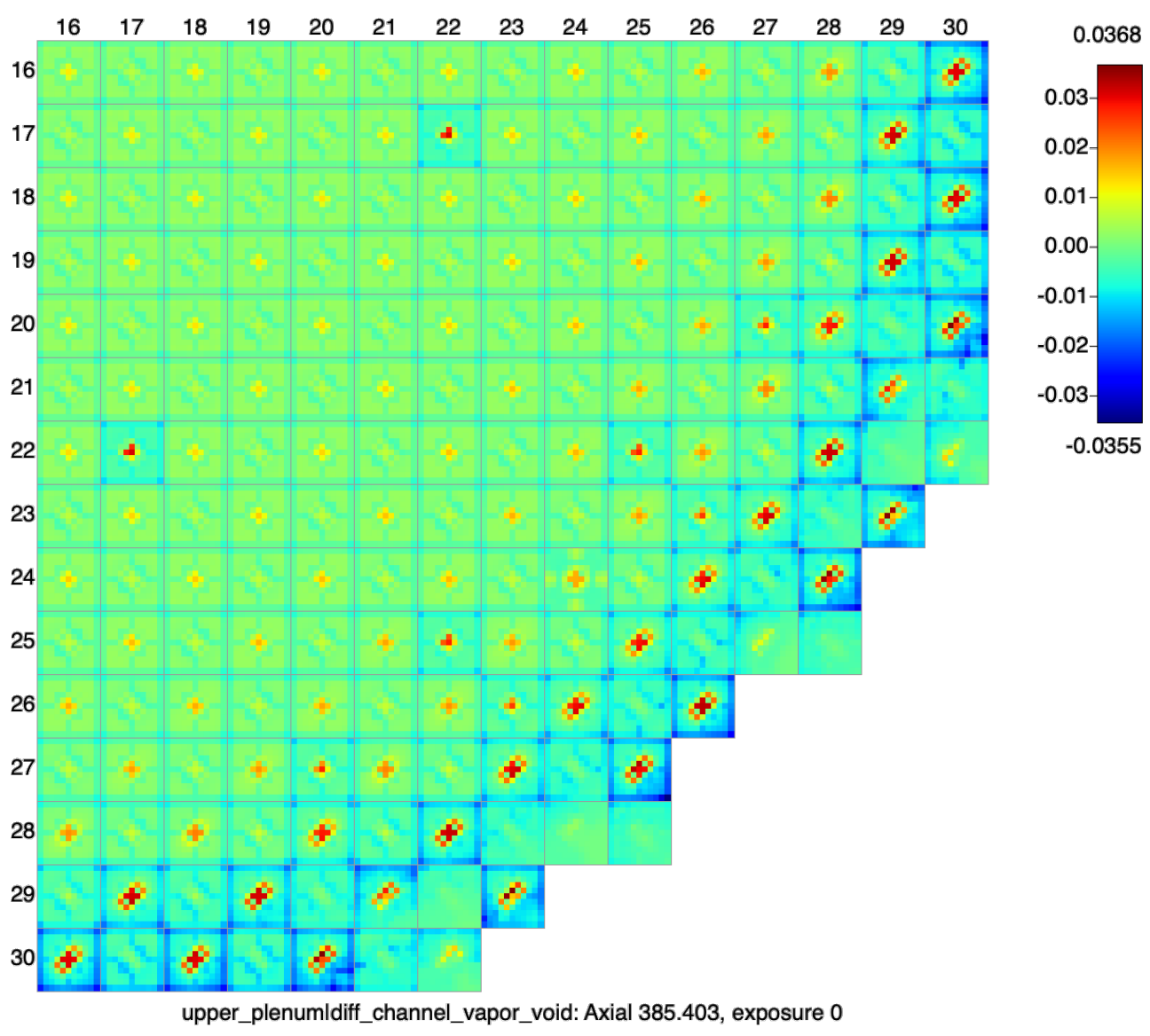

Figure 11. The difference in the outlet void distribution between a model of Peach Bottom with the upper plenum and one without the upper plenum.

The largest void differences occurred at the last heated plane in the model. Results for this plane are shown in Fig. 11. Void appears to concentrate around the large water rods. The maximum difference between the two cases was roughly $4 \%$ void.

After the bounds in the void plot were adjusted to be $\pm 1 \%$ and the axial level shown in Fig. 10 was considered, the void increased slightly in the inner region and decreased in the outer ring, which accounts for the power difference trend that was observed. These results are shown in Fig. 12.

To add to the trend analysis, the inlet mass flux differences are shown in Fig. 13. Consistent with the previous findings, the flow was more restricted to the inner region of the core, whereas it was higher in the peripheral region. This will lead to higher void in the inner region and lower void in the periphery region.

Finally, the difference between the radial pressure distribution at the model inlet is shown in Fig. 14. The difference is always positive because including the upper plenum results in an additional pressure drop in all assemblies. However, the pressure drop increase in the inner region of the core was higher than in the peripheral region. 


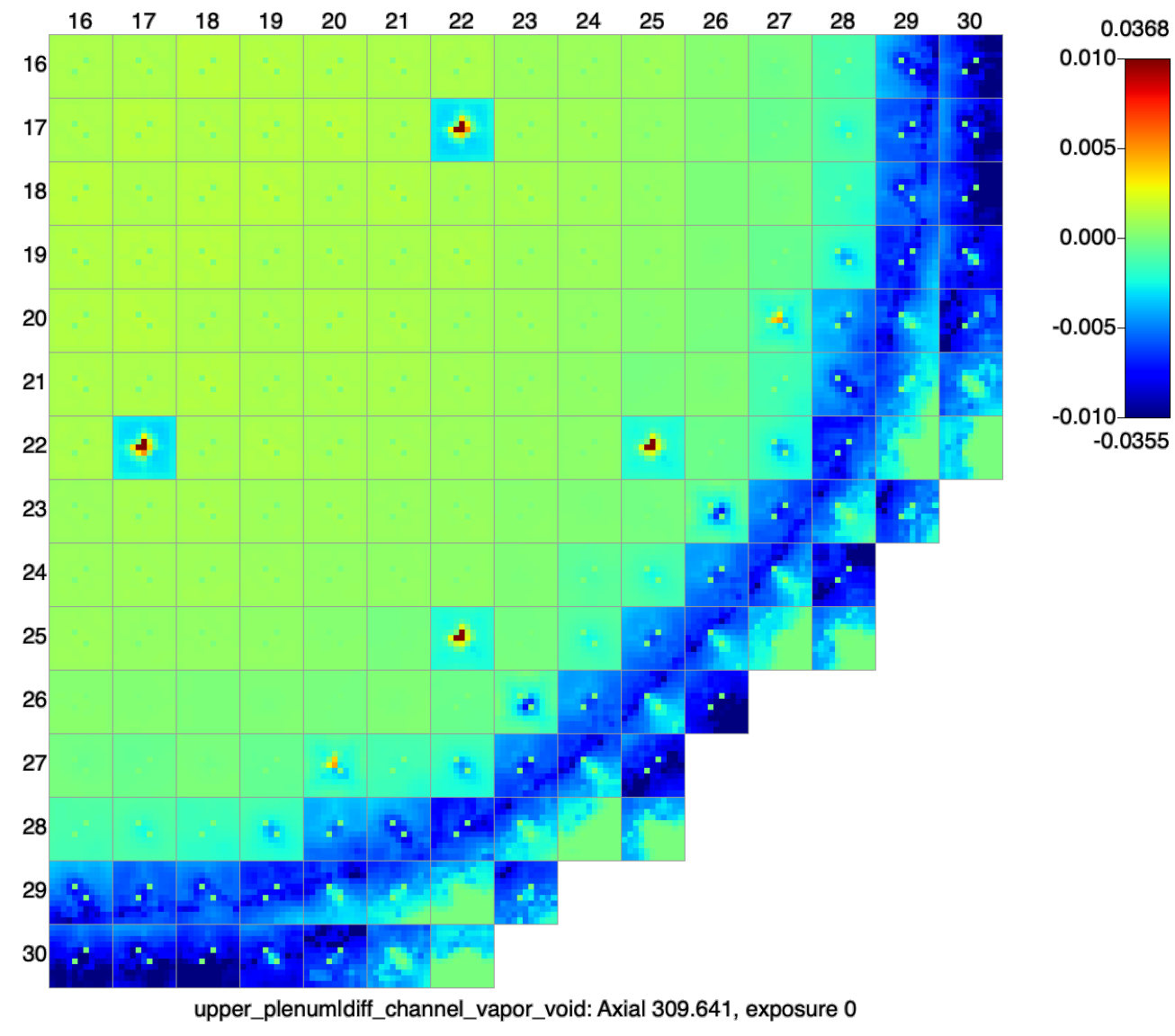

Figure 12. The difference in the void distribution between a model of Peach Bottom with the upper plenum and one without the upper plenum. Results are shown for the $309.64 \mathrm{~cm}$ axial location. 


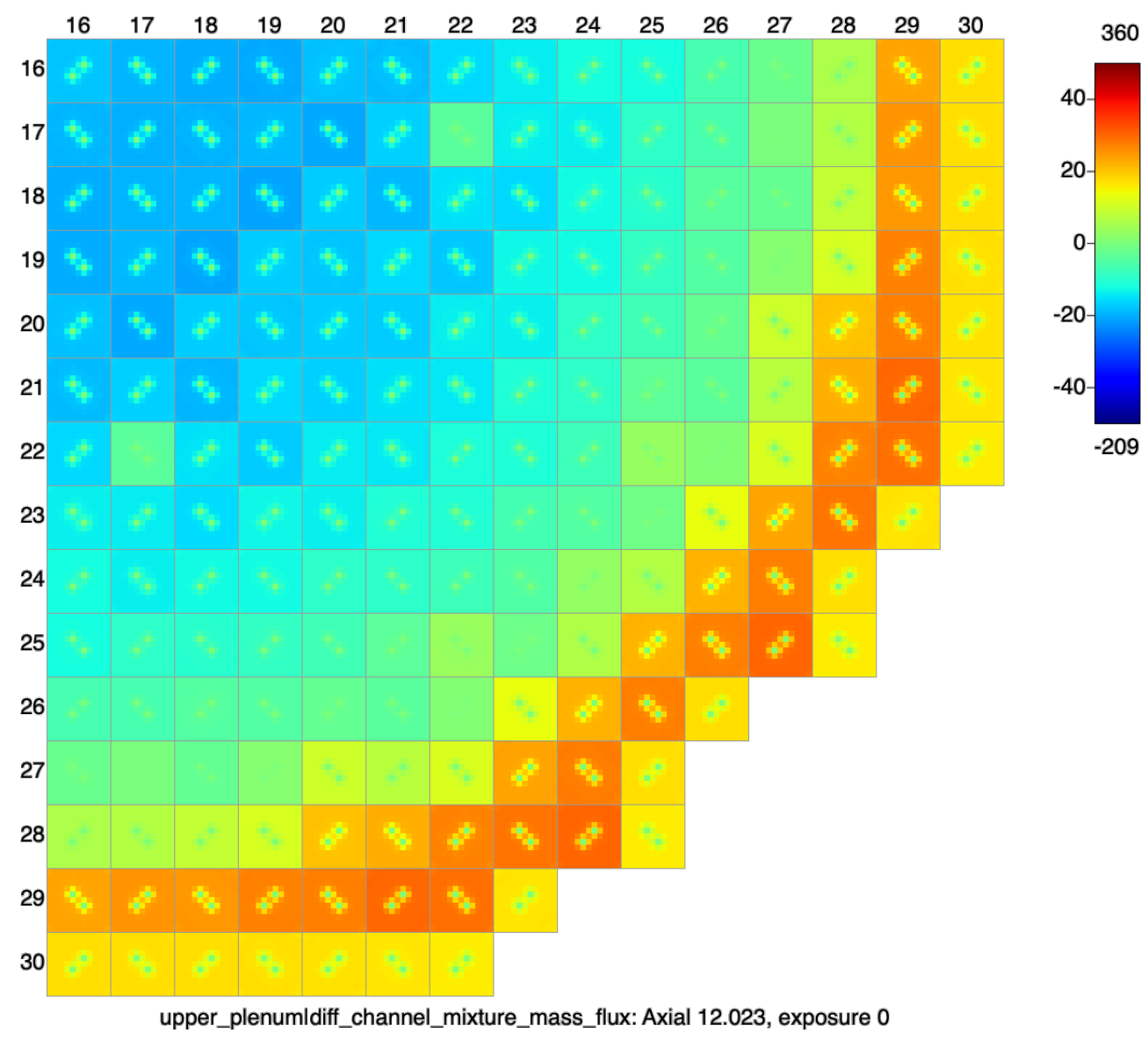

Figure 13. The difference in the inlet mass flux distribution between a model of Peach Bottom with the upper plenum and one without the upper plenum. 


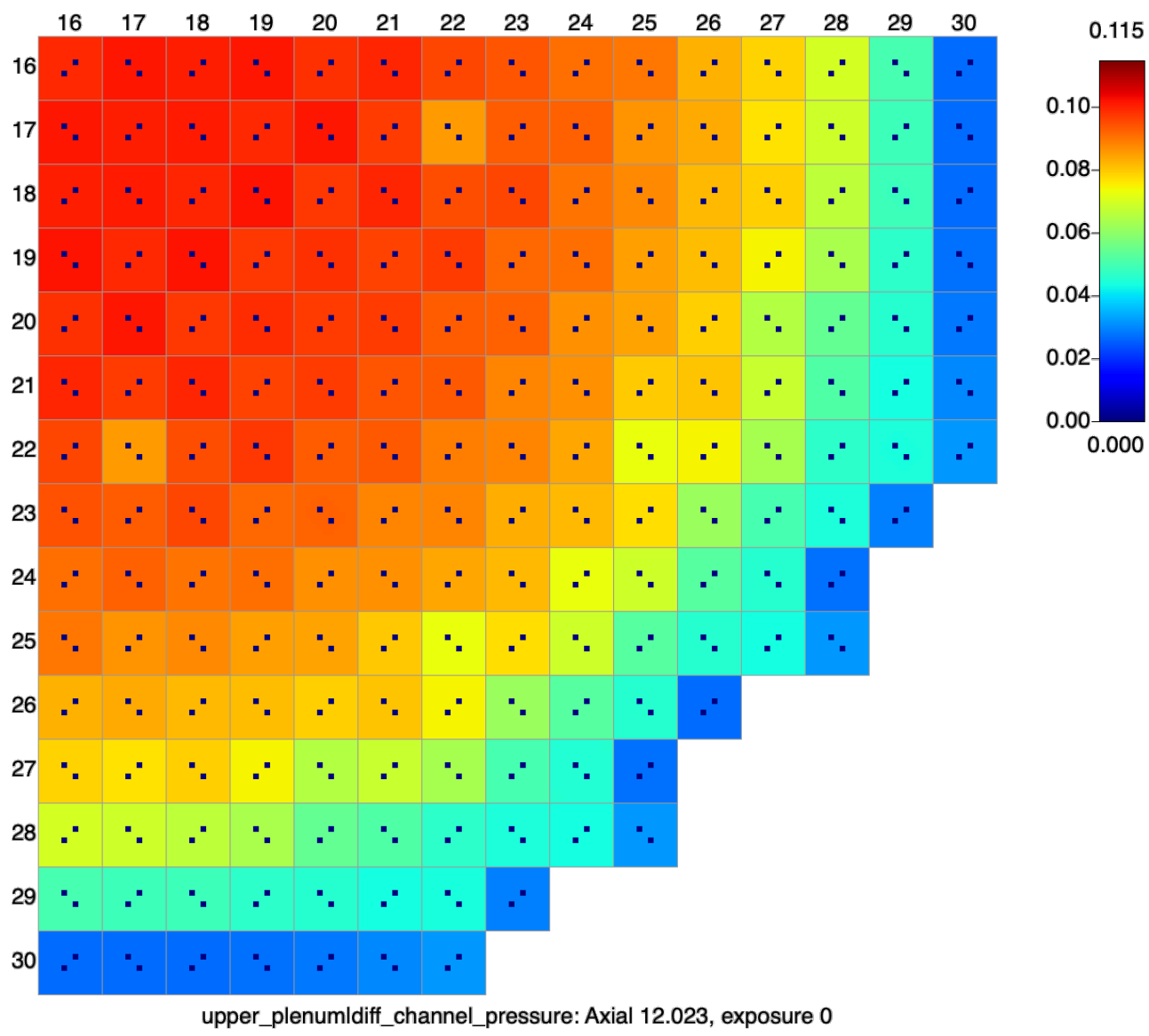

Figure 14. The difference in the inlet pressure distribution between a model of Peach Bottom with the upper plenum and one without the upper plenum. 


\subsection{GRID LOSS TREATMENT REVIEW}

The spacer grids (SGs) play an important role in the fuel rods of both PWR and BWR cores. From a mechanical point of view, they help maintain the rod spacing and the structural integrity of the fuel assembly by preventing possible flow-induced vibrations and rod bowing. From a heat transfer point of view, their presence is expected to increase the turbulence level downstream, which is beneficial for both single- and two-phase flow mixing and heat transfer that can improve the departure-from-nucleate-boiling ratio for PWRs. It is also expected to improve critical power ratio (CPR) performance by increasing the thickness of film in the annular flow regime in the top section of the reactor cores of BWRs. However, the disadvantage is that they create additional pressure loss within the system, thus requiring a higher pump power to provide adequate pressure head to drive the coolant through the entire system. The additional pressure drop increases the overall power plant operation cost and may pose significant safety concerns in certain accident core cooling scenarios where natural circulation is important. As a result, accurate quantification of SG-induced pressure drop is one of the crucial tasks performed for SG designs as well as for the reactor $\mathrm{T} / \mathrm{H}$ analysis under normal operation and abnormal transients. The goal of this study is to assess the CTF grid loss model and make recommendations to improve the code's predictive capabilities. Before the newly proposed grid model is detailed, an overview of the current CTF model is given. CTF uses a conventional approach for the pressure drop across an obstacle to correlate the pressure drop as a velocity head with a loss coefficient, as shown in the following equation:

$$
\Delta P=\frac{1}{2} \zeta \alpha_{k} \rho_{k} u_{k}^{2}
$$

where $\zeta$ is the pressure loss coefficient, $\rho$ is the density, and $u$ is the flow velocity in the vertical direction of phase $k$. The pressure loss coefficients are defined assuming positive upflow in a channel and specified for a momentum (not continuity) cell containing the obstruction. Close scrutiny of the current CTF spacer loss model expressed by Eq. (28) reveals that the model lacks a two-phase flow multiplier to capture the two-phase flow hydraulic behavior involved in PWRs under abnormal transients and in BWRs under normal and/or abnormal operation conditions. In addition, the effects of the spacer on the droplet pressure drop are not considered. To improve the CTF predictive capability, the team proposes the same general format correlations developed for the two-phase flow frictional pressure drop: that is, using the single-phase pressure loss coefficient to calculate the two-phase pressure drop by introducing a two-phase multiplier of various forms:

$$
\Delta P=\zeta \frac{G^{2}}{2 \rho_{l}} \Phi_{h o m}^{2} \Phi_{c o r r}^{2},
$$

where $G$ is the total mass flux defined as $G=\left(1-\alpha_{v}\right) \rho_{\ell} u_{l}+\rho_{v} \alpha_{v} u_{v}$ and $\Phi_{h o m}$ is the homogeneous two-phase flow multiplier given by

$$
\Phi_{h o m}^{2}=\left(1+x\left(\frac{\rho_{\ell}}{\rho_{v}}-1\right)\right),
$$

and $\Phi_{\text {corr }}$ is a correction two-phase flow multiplier of Chisholm type given by

$$
\Phi_{\text {corr }}^{2}=a x(1-x)^{b}+c x^{e}
$$

where $x$ is the quality and $a, b, c$, and $d$ are coefficients that can be calibrated using experimental data to match the two-phase pressure drop. $\zeta$ is the pressure loss coefficient, which is usually determined from 
experiments as a function of the liquid Reynolds number. To capture this effect, a correction to $\zeta$ function of the liquid Reynolds number can be used in the form of:

$$
\zeta_{\text {corr }}=\zeta\left(f+g R e_{\ell}^{h}\right)
$$

where $R e_{\ell}$ is the liquid Reynolds number, and $f, g$, and $h$ are constants calibrated from data.

It is important to note that these models cannot be universally applied outside of the original experimental conditions under which they were developed. This is because pressure loss coefficients are highly geometry dependent. For each spacer type, single- and two-phase pressure drop data are needed to calibrate the model. The current proposed model can be readily implemented into existing $\mathrm{T} / \mathrm{H}$ analysis codes to further improve their prediction.

\subsection{LOWER TIE PLATE FORM LOSS}

In previous work, a feature was added for capturing the inlet orifice loss coefficient. The inlet orifice is a part of the lower core plate and does not move during a fuel shuffle. This form loss is important to capture because it affects the core flow distribution, which impacts power and void generation in individual bundles. Another important inlet form loss is the tie-plate loss coefficient, which is part of the bundle itself and moves with the assembly during a fuel shuffle. To capture this form loss, the VERAIO input file was modified to allow the user to specify a form loss for the assembly's lower nozzle. The input was made optional, so if it is omitted, there will be no form loss associated with the assembly inlet. In addition to the form loss coefficient, an optional input was added for the nozzle's reference flow area. Both of these inputs will be provided by the fuel vendor.

These new inputs are passed to CTF through the Xml2ctf preprocessor and the CTF input file. The loss is only used for BWR models, because these models are applied in the pressure balance iteration loop, which is responsible for balancing the flow to the assemblies so that the pressure drops in all core assemblies are equal. The calculation of the lower tie plate pressure drop is performed as follows:

$$
\Delta p=\frac{1}{2} k \frac{\dot{m}^{2}}{\rho_{\ell} A^{2}}
$$

In the equation, $k$ and $A$ are the user-supplied form loss and reference area. If the area is not provided, then a default reference area of $10 \epsilon^{2}$ is used, which is a standard reference area. The mass flow rate into the assembly is $\dot{m}$, which will be calculated as part of the pressure balance iteration loop. Finally, $\Delta p$ is the pressure drop over the lower tie plate. The density of the liquid is represented by $\rho_{\ell}$. This pressure drop is added to the inlet orifice pressure drop.

To test the new feature, a simple 12-assembly BWR model was created. The assemblies in the model were identical, except the peripheral assemblies had a lower tie plate form loss applied. A diagram of the core assembly layout is shown below as follows: the " 0 " values represent blank spots (no assembly), "1" indicates a peripheral assembly with no tie plate form loss, and " 2 " indicates an inner assembly with a tie plate form loss. 
Table 6. Geometry details for the lower tie plate form loss verification test

\begin{tabular}{rcc}
\hline Parameter & Periphery bundles & Inner bundles \\
\hline Flow area & $1.02 \times 10^{-2} \mathrm{~m}$ \\
Dh & $1.36 \times 10^{-2} \mathrm{~m}$ \\
$\mathrm{~L}$ & $3.658 \mathrm{~m}$ \\
Nozzle form loss & 0.0 & 10.0 \\
Nozzle area & $3 \times 10^{-3} \mathrm{~m}$ & $6.45 \times 10^{-3} \mathrm{~m}$ \\
Orifice form loss & 1.0 & 1.0 \\
Orifice area & $6.45 \times 10^{-3} \mathrm{~m}$ \\
\hline
\end{tabular}

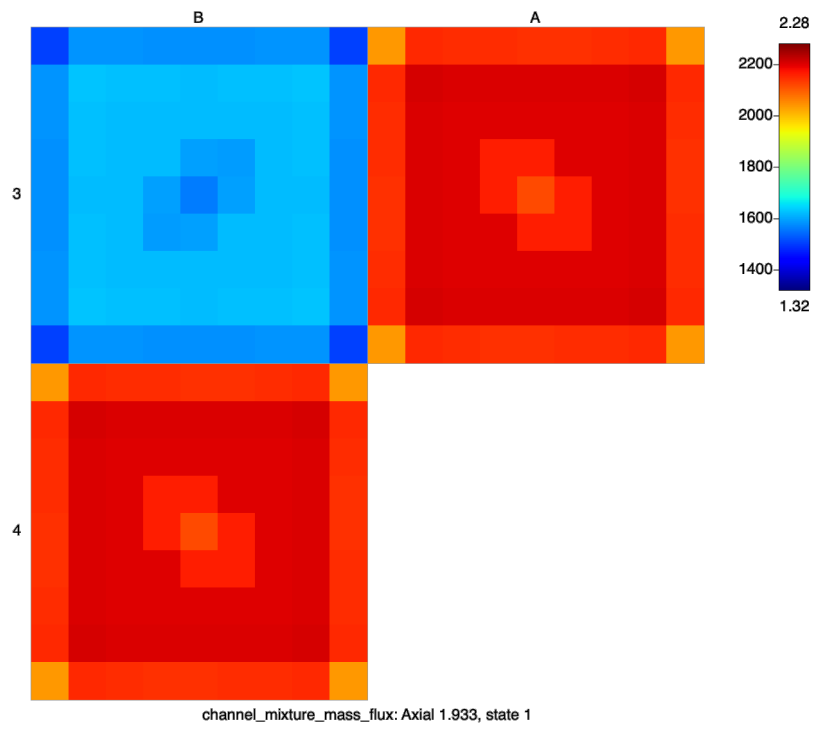

Figure 15. Calculated flow distribution in the first level of the tie plate verification test.

0110

1221

1221

0110

The case was modeled in quarter symmetry, the power was set to zero, and all spacer grid form losses were removed to make it easier to calculate an analytical solution. A constant friction factor of 0.1 was applied (no Reynolds dependence). An analytical solution was performed considering the details shown in Table 6. The flow distribution in the first level of the solution is shown in Fig. 15.

The inlet mass flow rate to the inner assembly, as calculated by CTF, was $16.4 \mathrm{~kg} \mathrm{~s}^{-1}$, and the inlet mass flow rate to the outer assembly was $22.3 \mathrm{~kg} \mathrm{~s}^{-1}$. Using these values, along with the geometry values from Table 6, leads to the pressure drop breakdown shown in Table 7.

The error between the bundle pressure drops is $0.8 \%$, or roughly 0.1 psi. Note that the converged solution results in bundle pressure differences of up to $0.36 \mathrm{psi}$ as a result of the tolerance set in the pressure balance 
Table 7. Analytical calculation of the pressure drop breakdown in the lower tie plate verification test

\begin{tabular}{lrr}
\hline & Periphery bundles [Pa] & Inner bundles [Pa] \\
\hline Friction & $4.60 \cdot 10^{4}$ & $8.59 \cdot 10^{4}$ \\
Tie plate & $4.28 \cdot 10^{4}$ & $0.00 \cdot 10^{0}$ \\
Orifice & $7.99 \cdot 10^{3}$ & $4.28 \cdot 10^{3}$ \\
\hline Total & $9.39 \cdot 10^{4}$ & $9.31 \cdot 10^{4}$ \\
\hline
\end{tabular}

loop. Therefore, this is considered excellent agreement with the analytical solution. 


\section{SUMMARY}

This report summarizes several geometry modeling and numerical improvements to CTF that were implemented during the first half of this fiscal year. These improvements are part of a larger plan to develop CTF into a state-of-the-art BWR modeling and simulation tool. The geometry modeling improvements will now allow CTF to model cores with mixed fuel designs, as well as to model the upper plenum region of the core. Additionally, the spacer grid loss model was reviewed, and it was determined that a two-phase correction must be implemented to correctly capture the spacer grid pressure loss in two-phase models.

The numerical improvements were primarily focused on improving CTF performance, both in terms of stability and runtime. The parallelization was improved by allowing CTF to use more processors in the solution, which leads to a roughly $1.6 \times$ speedup of the total VERA simulation runtime for a Peach Bottom Unit 2 steady state case. Despite this improvement, CTF still accounts for roughly $80 \%$ of the total VERA runtime. For that reason, a more aggressive set of changes is planned, which involves making the governing equation solution more implicit, so that much larger timesteps can be taken. This report outlines this planned set of improvements . Although some of the changes have been implemented, more work is needed to realize the full benefit of the more implicit solution. 



\section{REFERENCES}

R. Salko and V. Kumar. Assessment of CTF Needs for Modeling of Boiling Water Reactors. Technical Report ORNL/TM-2020-3, Oak Ridge National Laboratory, 2020.

R. Salko, A. Wysocki, M. Avramova, A. Toptan, N. Porter, T. Blyth, C. Dances, A. Gomez, C. Jernigan, and J. Kelly. CTF Theory Manual. The North Carolina State University, 2017.

R. Salko, B. Hizoum, B. Collins, and M. Asgari. Improvements to CTF for modeling of Boiling Water Reactor Geometry and Operating Conditions. Technical Report ORNL/TM-2020/1746, Oak Ridge National Laboratory, 2020a.

R. Salko, V. Kumar, and B. Hizoum. Improvements to CTF Closure Models for Modeling of Two-Phase Flow. Technical Report ORNL/TM-2020/3, Oak Ridge National Laboratory, 2020 b. 